\title{
Supporting Infromation for
}

1,8-Naphthalimide-based highly emissive luminophors with various mechanofluorochromism and aggregation-induced characteristics

\author{
Ya Yin, Zhao Chen*, Congbin Fan, Gang Liu, Shouzhi Pu* \\ Jiangxi Key Laboratory of Organic Chemistry, Jiangxi Science and Technology Normal \\ University, Nanchang 330013, P. R. China
}

\begin{abstract}
Corresponding Author
*E-mail address: chenzhao666@126.com (Z.Chen), pushouzhi@tsinghua.org.cn (S.Pu)

Tel: +86-791-83831996 Fax: +86-791-83831996
\end{abstract}

Table of Contents

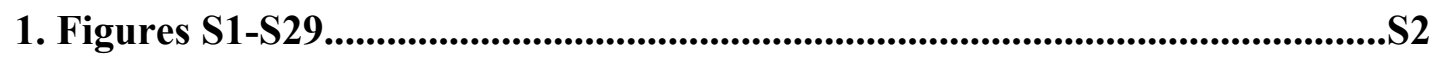

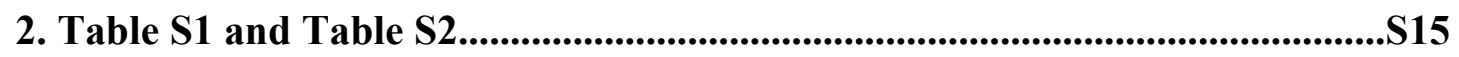

3. Copies of NMR spectra and Mass spectra...................................................S19 


\section{Figures S1-S29}

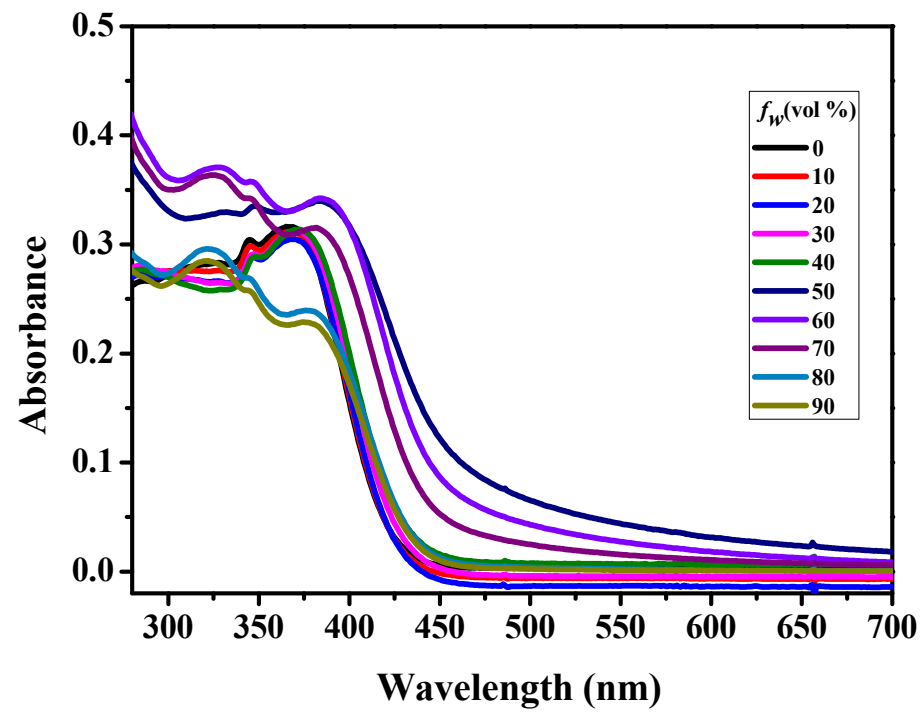

Figure S1. UV-Vis absorption spectra of compound 1 (concentration: $20 \mu \mathrm{M}$ ) in DMF-water mixtures with different volume fractions of water $(0-90 \%)$.

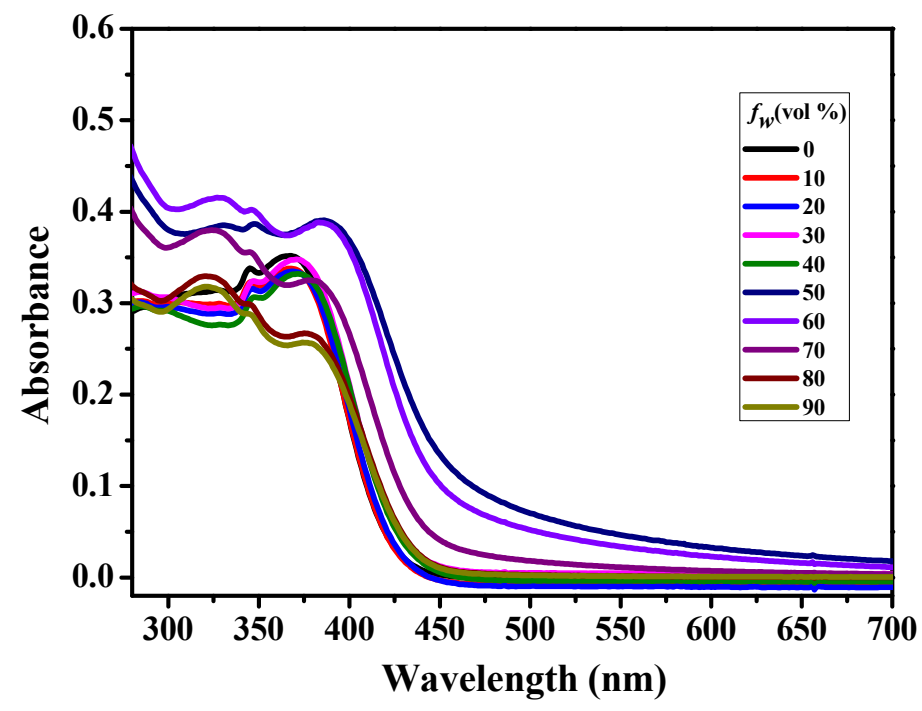

Figure S2. UV-Vis absorption spectra of compound 2 (concentration: $20 \mu \mathrm{M}$ ) in DMF-water mixtures with different volume fractions of water $(0-90 \%)$. 


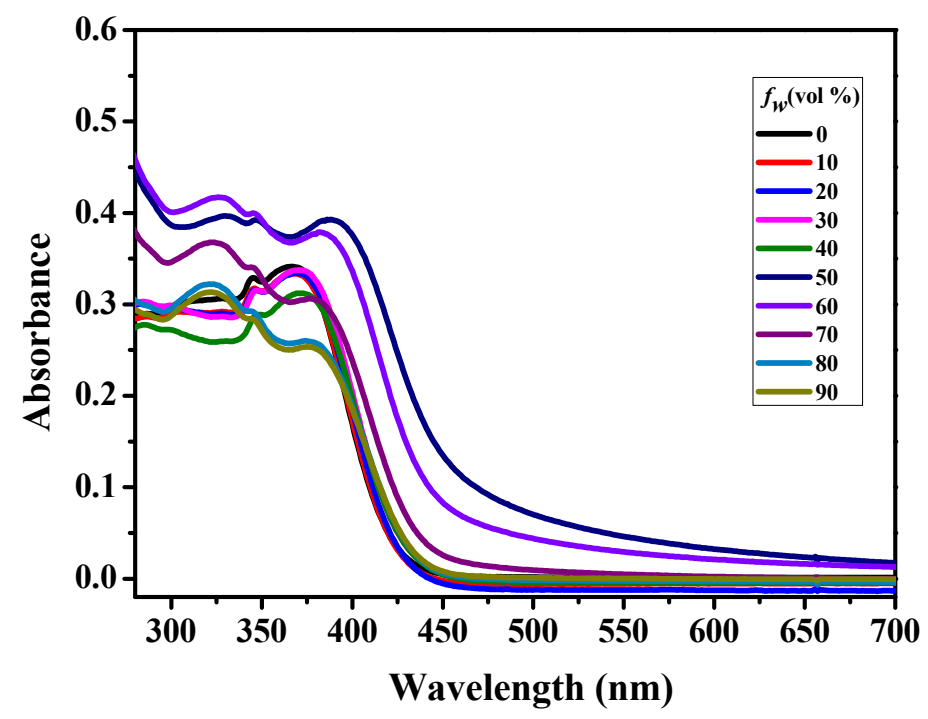

Figure S3. UV-Vis absorption spectra of compound 3 (concentration: $20 \mu \mathrm{M}$ ) in DMF-water mixtures with different volume fractions of water $(0-90 \%)$.

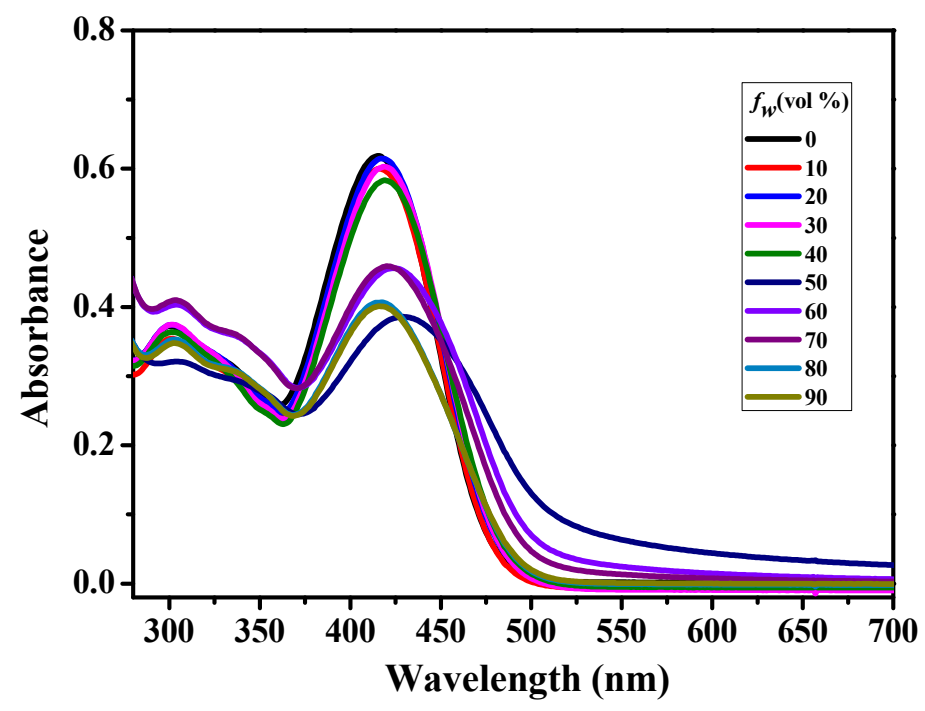

Figure S4. UV-Vis absorption spectra of compound 4 (concentration: $20 \mu \mathrm{M}$ ) in DMF-water mixtures with different volume fractions of water $(0-90 \%)$. 


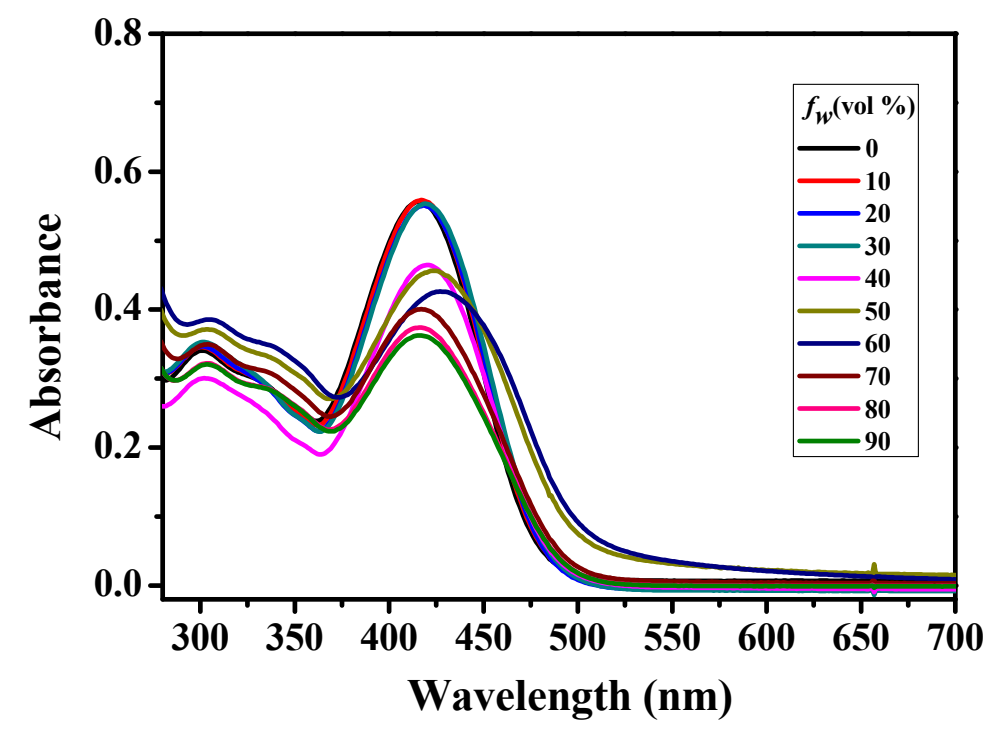

Figure S5. UV-Vis absorption spectra of compound 5 (concentration: $20 \mu \mathrm{M}$ ) in DMF-water mixtures with different volume fractions of water $(0-90 \%)$.

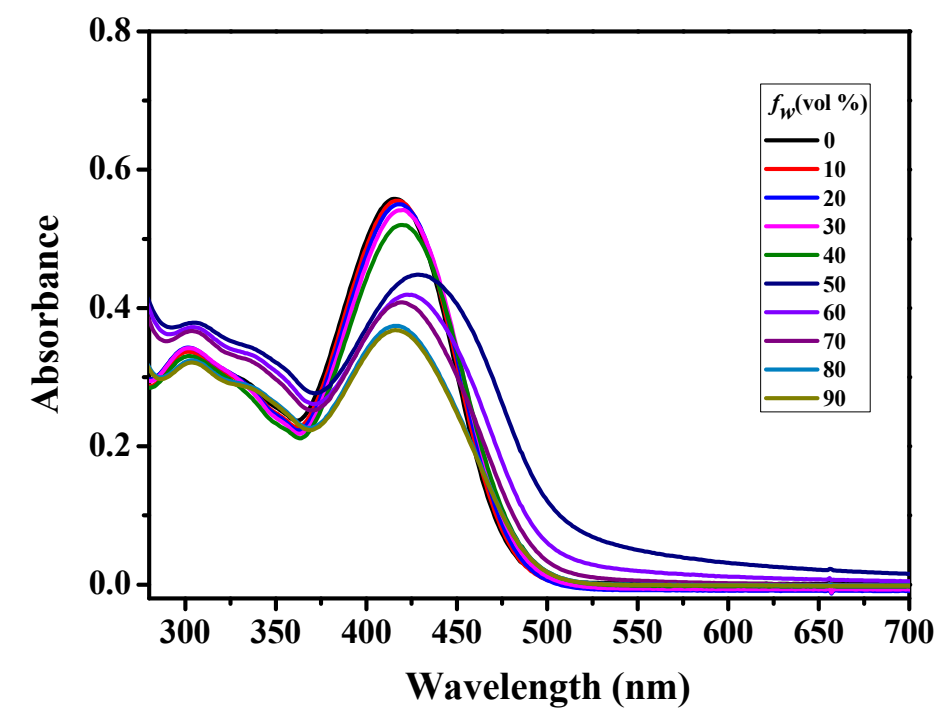

Figure S6. UV-Vis absorption spectra of compound 6 (concentration: $20 \mu \mathrm{M}$ ) in DMF-water mixtures with different volume fractions of water (0-90\%). 

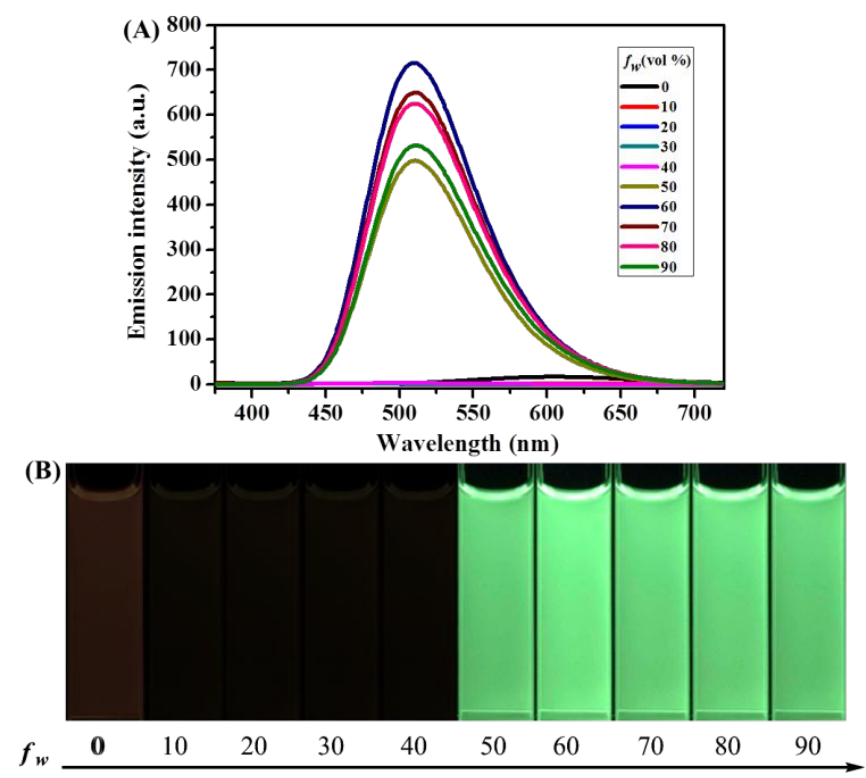

Figure S7. (A) PL spectra of the dilute solutions of luminogen 2 (concentration: $20 \mu \mathrm{M}$ ) in DMF- $\mathrm{H}_{2} \mathrm{O}$ mixtures with different volume fractions of water (0-90\%). Excitation wavelength: $365 \mathrm{~nm}$; (B) PL images of luminogen 2 (concentration: $20 \mu \mathrm{M}$ ) in DMF- $\mathrm{H}_{2} \mathrm{O}$ mixtures with different $f_{\mathrm{w}}$ values $(0-90 \%)$ under irradiation with UV light at $365 \mathrm{~nm}$.
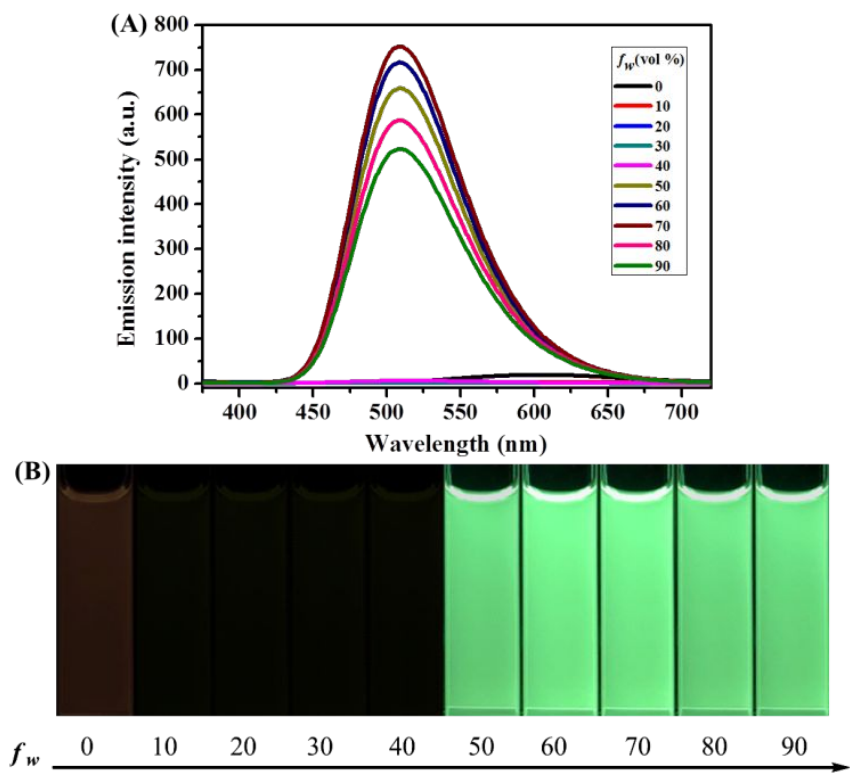

Figure S8. (A) PL spectra of the dilute solutions of luminogen 3 (concentration: $20 \mu \mathrm{M}$ ) in DMF- $\mathrm{H}_{2} \mathrm{O}$ mixtures with different volume fractions of water $(0-90 \%)$. Excitation wavelength: $365 \mathrm{~nm}$; (B) PL images of luminogen 3 (concentration: $20 \mu \mathrm{M}$ ) in DMF- $\mathrm{H}_{2} \mathrm{O}$ 
mixtures with different $f_{\mathrm{w}}$ values (0-90\%) under irradiation with UV light at $365 \mathrm{~nm}$.
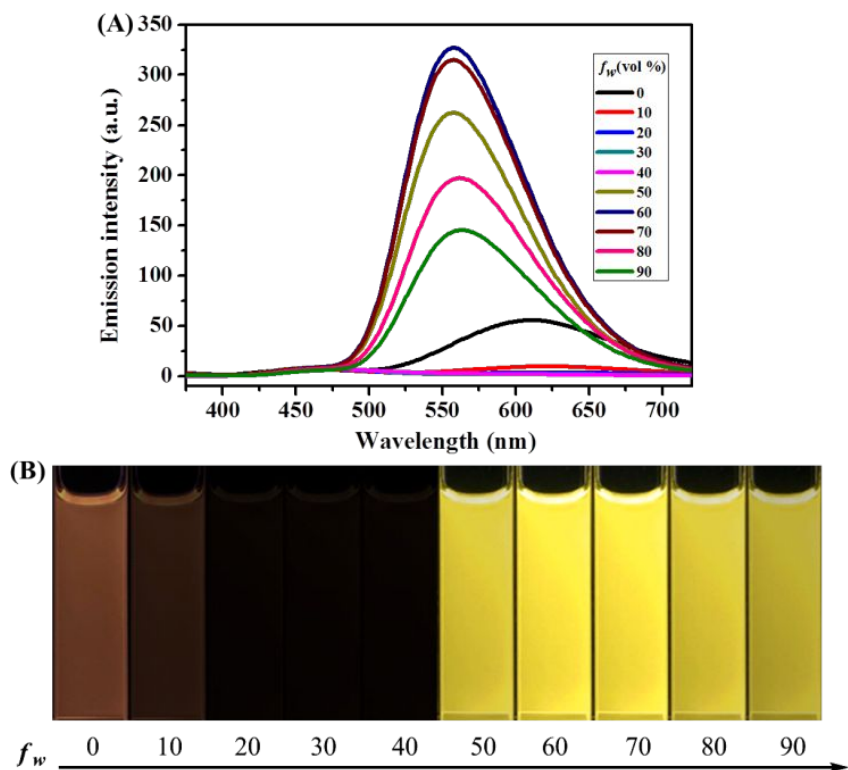

Figure S9. (A) PL spectra of the dilute solutions of luminogen 4 (concentration: $20 \mu \mathrm{M}$ ) in DMF- $\mathrm{H}_{2} \mathrm{O}$ mixtures with different volume fractions of water $(0-90 \%)$. Excitation wavelength: $365 \mathrm{~nm}$; (B) PL images of luminogen 4 (concentration: $20 \mu \mathrm{M}$ ) in DMF- $\mathrm{H}_{2} \mathrm{O}$ mixtures with different $f_{\mathrm{w}}$ values (0-90\%) under irradiation with UV light at $365 \mathrm{~nm}$.
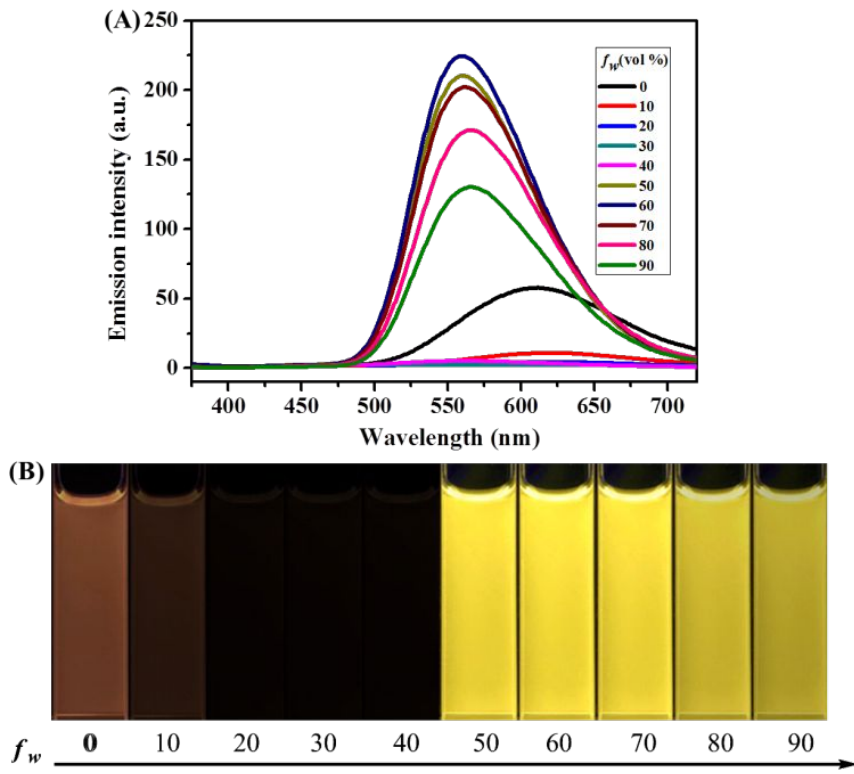

Figure S10. (A) PL spectra of the dilute solutions of luminogen 5 (concentration: $20 \mu \mathrm{M}$ ) in DMF- $\mathrm{H}_{2} \mathrm{O}$ mixtures with different volume fractions of water (0-90\%). Excitation 
wavelength: $365 \mathrm{~nm}$; (B) PL images of luminogen 5 (concentration: $20 \mu \mathrm{M}$ ) in DMF- $\mathrm{H}_{2} \mathrm{O}$ mixtures with different $f_{\mathrm{w}}$ values (0-90\%) under irradiation with UV light at $365 \mathrm{~nm}$.
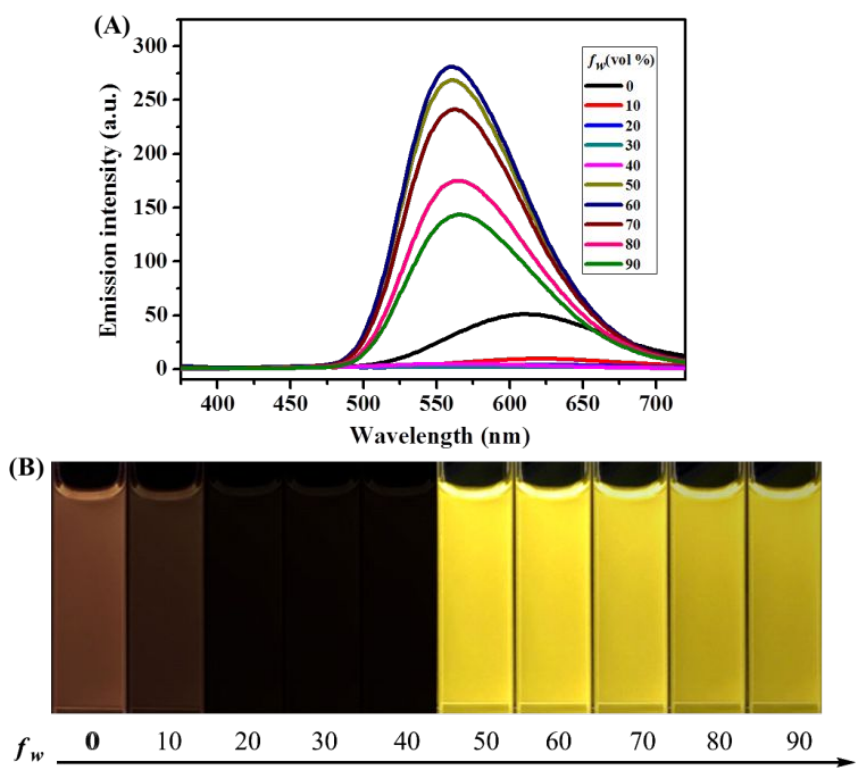

Figure S11. (A) PL spectra of the dilute solutions of luminogen 6 (concentration: $20 \mu \mathrm{M}$ ) in DMF- $\mathrm{H}_{2} \mathrm{O}$ mixtures with different volume fractions of water $(0-90 \%)$. Excitation wavelength: $365 \mathrm{~nm}$; (B) PL images of luminogen 6 (concentration: $20 \mu \mathrm{M}$ ) in DMF- $\mathrm{H}_{2} \mathrm{O}$ mixtures with different $f_{\mathrm{w}}$ values (0-90\%) under irradiation with UV light at $365 \mathrm{~nm}$.

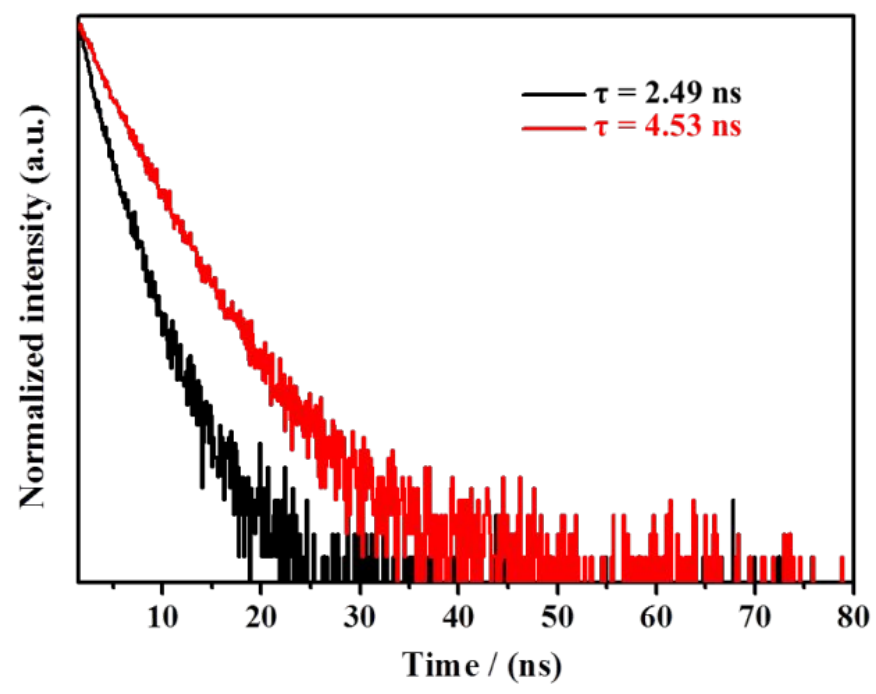


Figure S12. Decay curves (Excitation wavelength: $365 \mathrm{~nm}$ ) of luminogen 2 in various solid states: the unground solid sample (the black line, emission wavelength: $453 \mathrm{~nm}$ ) and the ground solid sample (the red line, emission wavelength: $508 \mathrm{~nm}$ ).

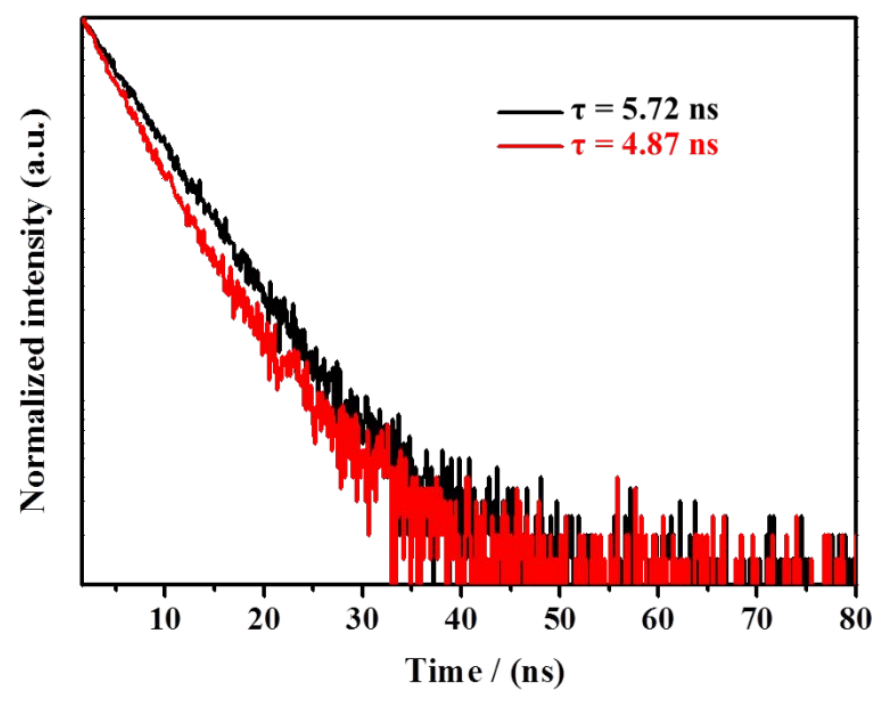

Figure S13. Decay curves (Excitation wavelength: $365 \mathrm{~nm}$ ) of luminogen 3 in various solid states: the unground solid sample (the black line, emission wavelength: $488 \mathrm{~nm}$ ) and the ground solid sample (the red line, emission wavelength: $507 \mathrm{~nm}$ ).

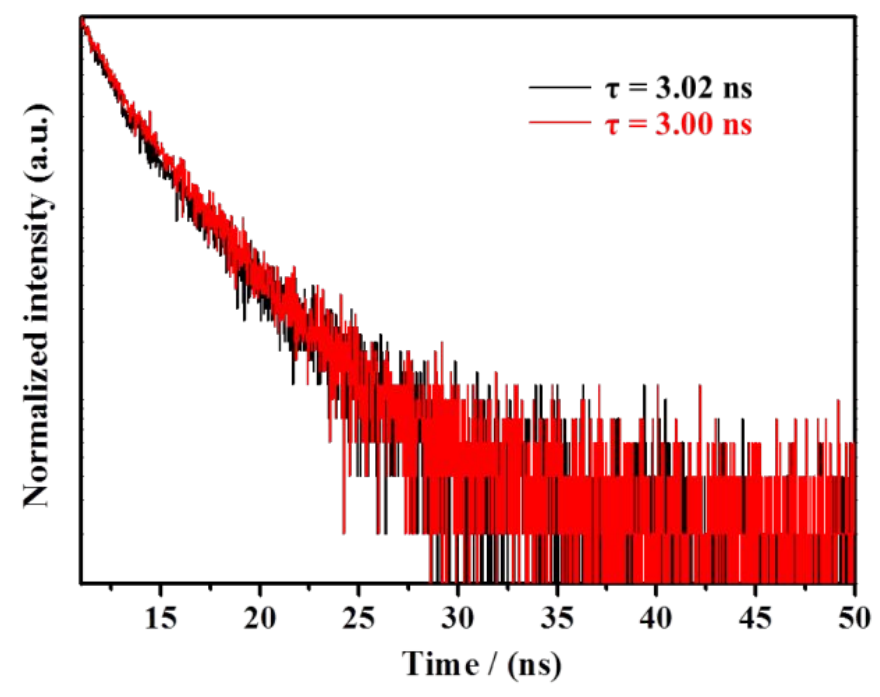


Figure S14. Decay curves (Excitation wavelength: $365 \mathrm{~nm}$ ) of luminogen 5 in various solid states: the unground solid sample (the black line, emission wavelength: $563 \mathrm{~nm}$ ) and the ground solid sample (the red line, emission wavelength: $572 \mathrm{~nm}$ ).

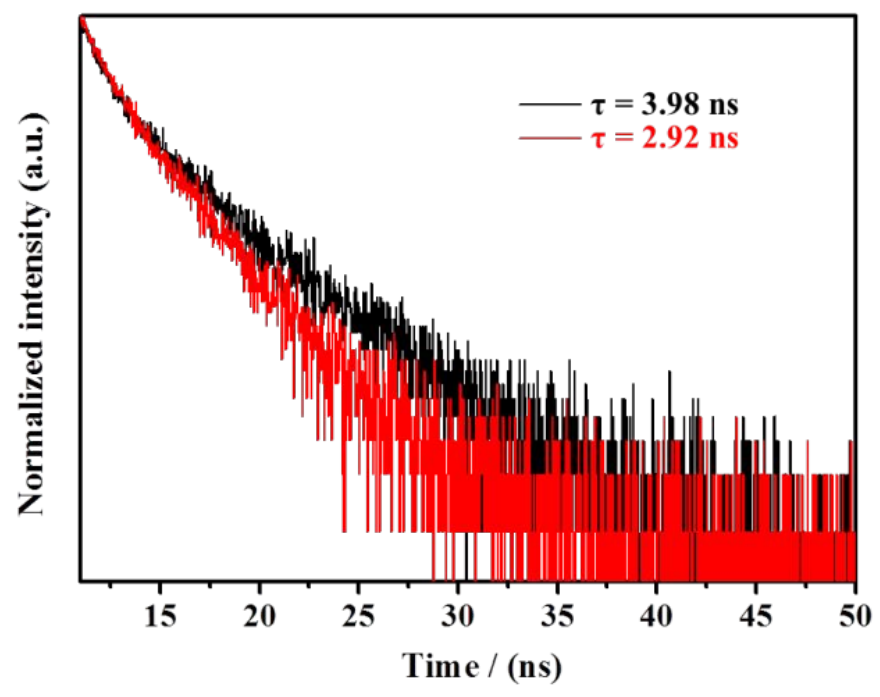

Figure S15. Decay curves (Excitation wavelength: $365 \mathrm{~nm}$ ) of luminogen 6 in various solid states: the unground solid sample (the black line, emission wavelength: $548 \mathrm{~nm}$ ) and the ground solid sample (the red line, emission wavelength: $566 \mathrm{~nm}$ ).

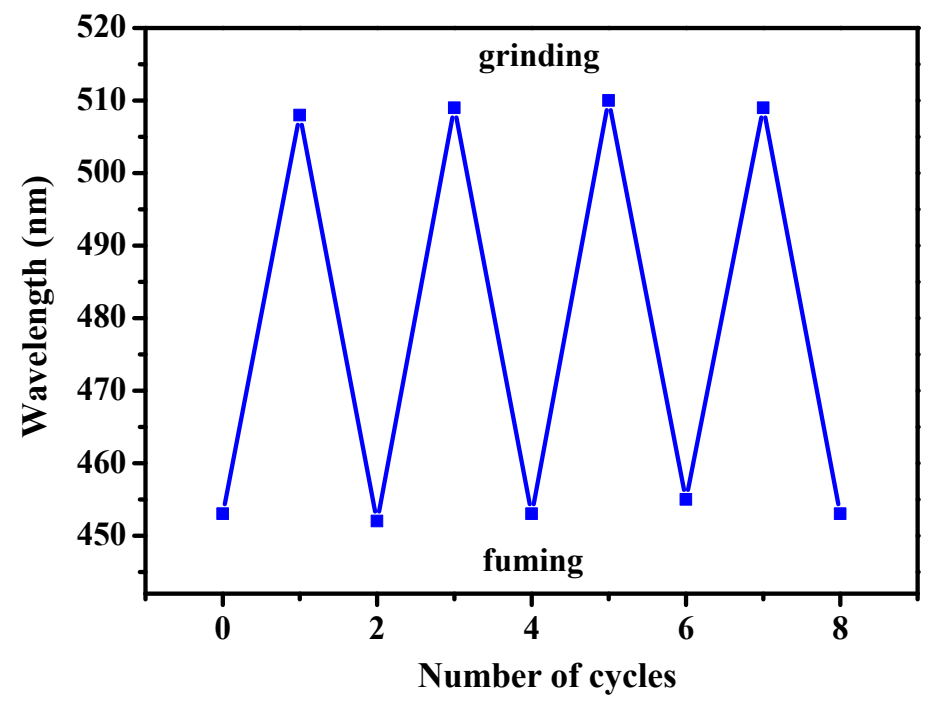


Figure S16. Repetitive experiment of mechanochromic behavior for luminogen 2 .

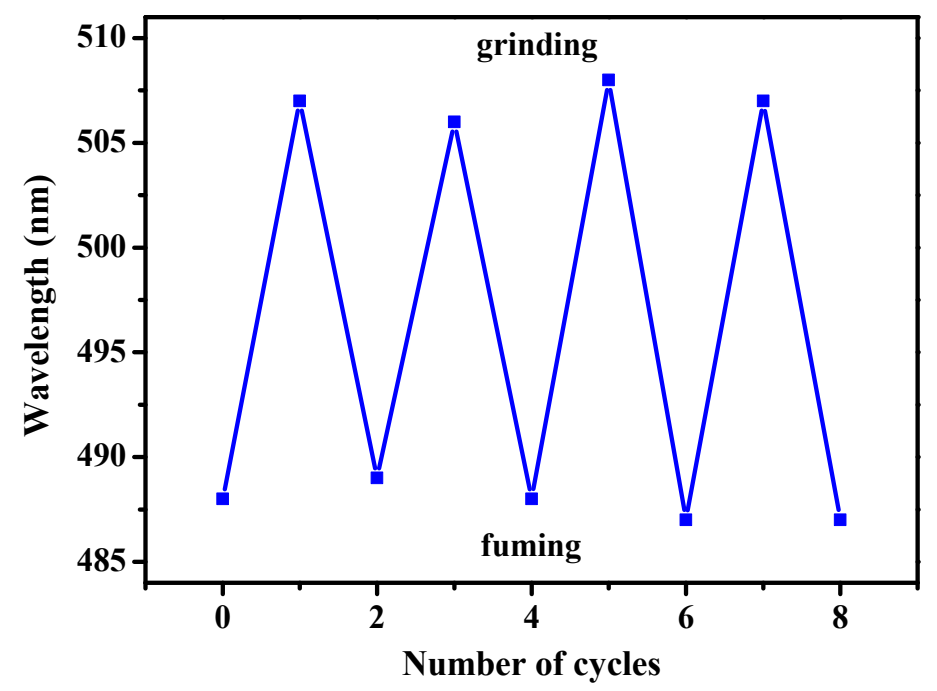

Figure S17. Repetitive experiment of mechanochromic behavior for luminogen 3.

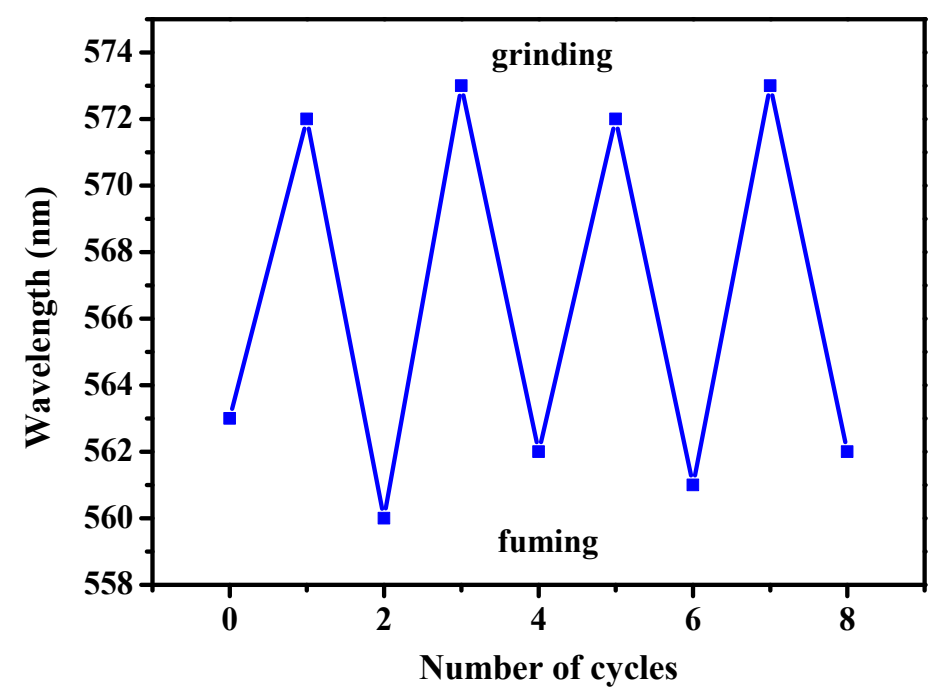

Figure S18. Repetitive experiment of mechanochromic behavior for luminogen 5. 


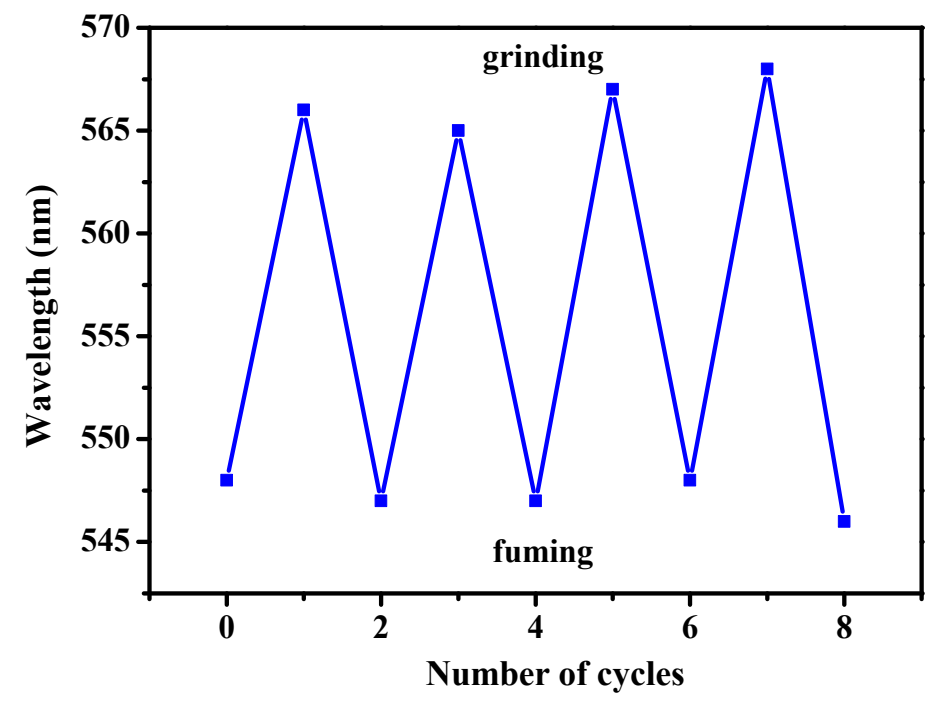

Figure S19. Repetitive experiment of mechanochromic behavior for luminogen 6.

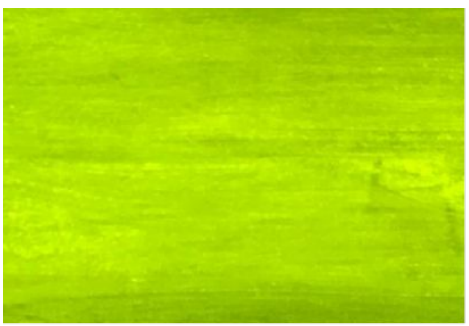

The initial state

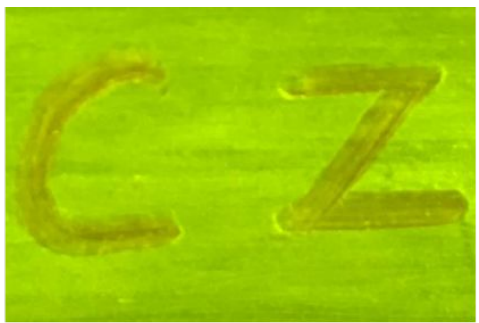

Writing

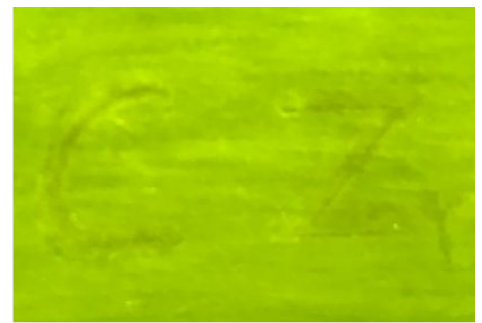

Fuming

Figure S20. Repeated writing and erasing processes utilizing the switchable mechanochromic fluorescence of $\mathbf{6}$.

\begin{tabular}{|c|c|c|}
\hline Compound & Solid States & $\operatorname{Em}_{\max }(\mathrm{nm})$ \\
\hline \multirow{4}{*}{1} & As-synthesized & 484 \\
\cline { 2 - 3 } & ground & 517 \\
\cline { 2 - 3 } & fumed & 485 \\
\hline \multirow{4}{*}{2} & As-synthesized & 453 \\
\cline { 2 - 3 } & ground & 508 \\
\cline { 2 - 3 } & fumed & 452 \\
\hline \multirow{3}{*}{3} & As-synthesized & 488 \\
\cline { 2 - 3 } & ground & 507 \\
\cline { 2 - 3 } & fumed & 489 \\
\cline { 2 - 3 } & As-synthesized & 562 \\
\cline { 2 - 3 } & ground & 568 \\
\hline \multirow{3}{*}{5} & fumed & 561 \\
\cline { 2 - 3 } & As-synthesized & 563 \\
\hline
\end{tabular}




\begin{tabular}{|c|c|c|}
\hline & fumed & 560 \\
\hline \multirow{3}{*}{6} & As-synthesized & 548 \\
\cline { 2 - 3 } & ground & 566 \\
\cline { 2 - 3 } & fumed & 547 \\
\hline
\end{tabular}

Figure S21. The maximum emission wavelengths of compounds 1-6 in different solid states.

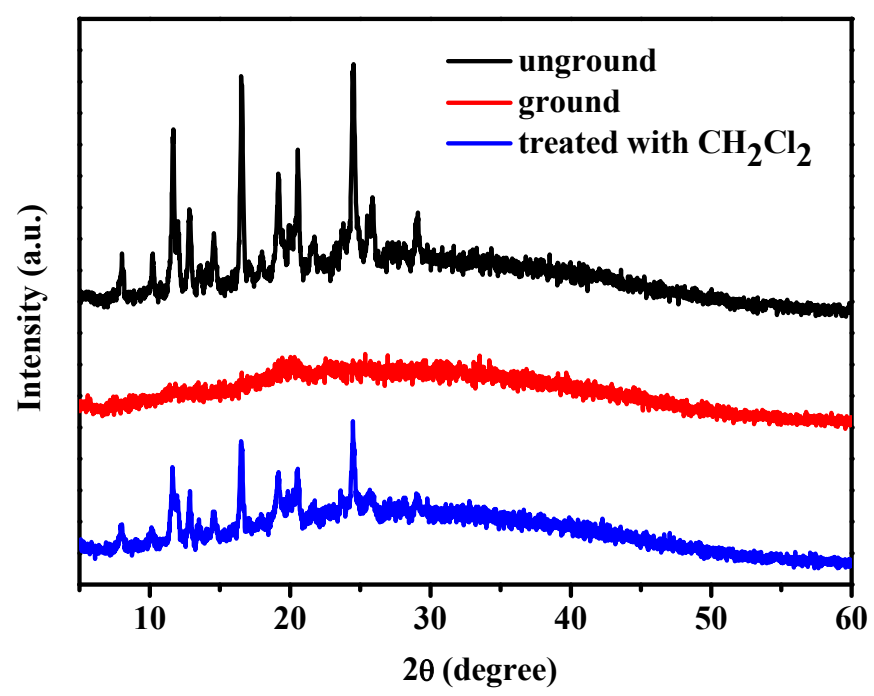

Figure S22. Powder XRD patterns of compound 2 in various solid states: the unground powder, the ground powder, the powder after treatment with dichloromethane vapor.

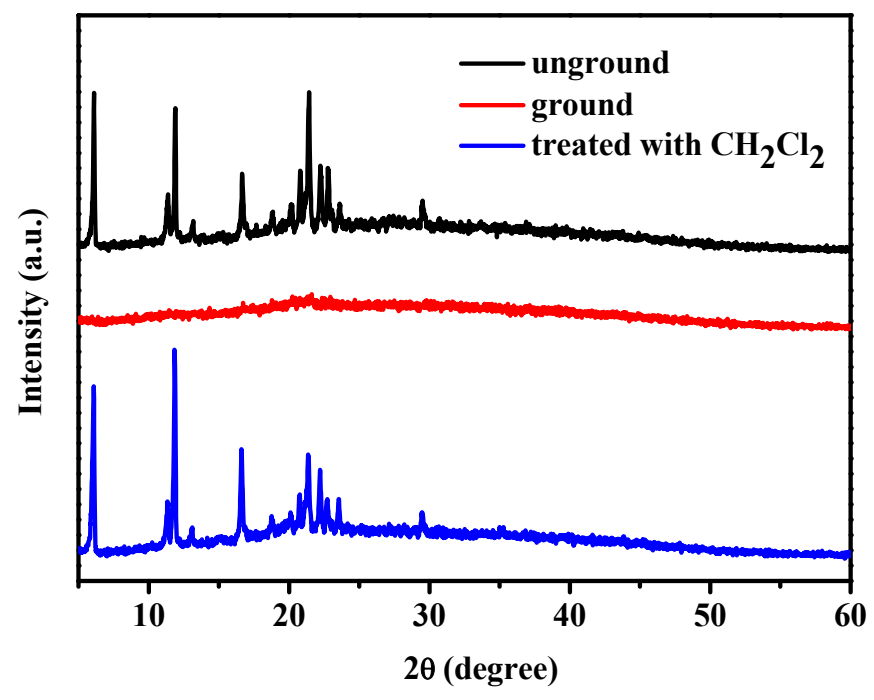


Figure S23. Powder XRD patterns of compound 3 in various solid states: the unground powder, the ground powder, the powder after treatment with dichloromethane vapor.

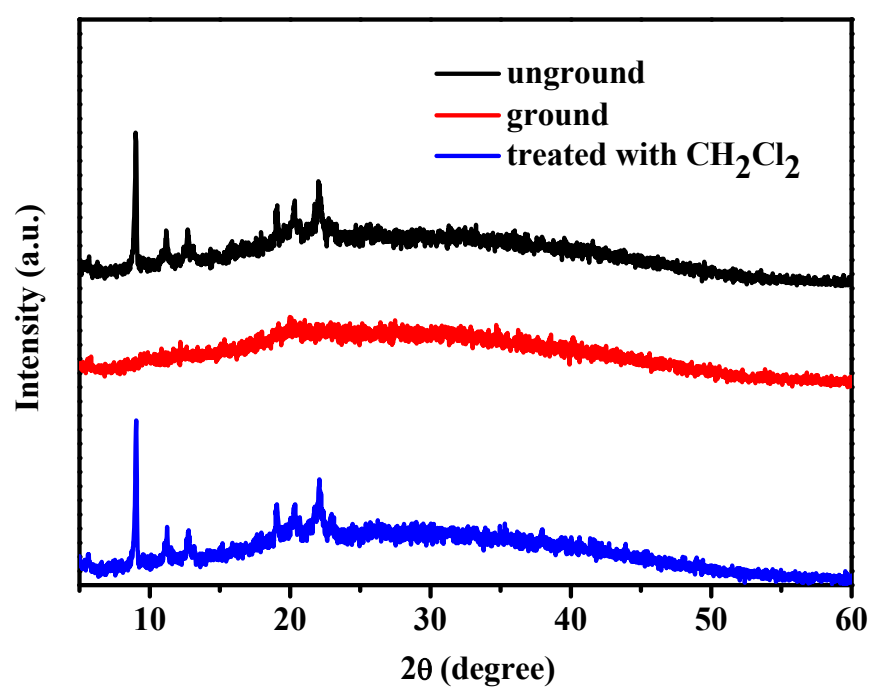

Figure S24. Powder XRD patterns of compound 4 in various solid states: the unground powder, the ground powder, the powder after treatment with dichloromethane vapor.

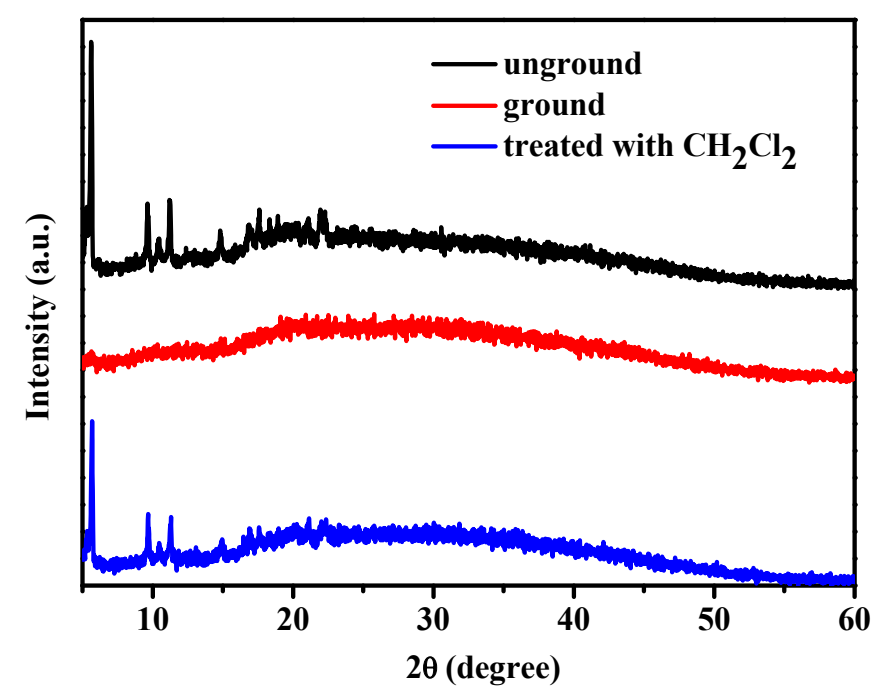

Figure S25. Powder XRD patterns of compound $\mathbf{5}$ in various solid states: the unground powder, the ground powder, the powder after treatment with dichloromethane vapor. 


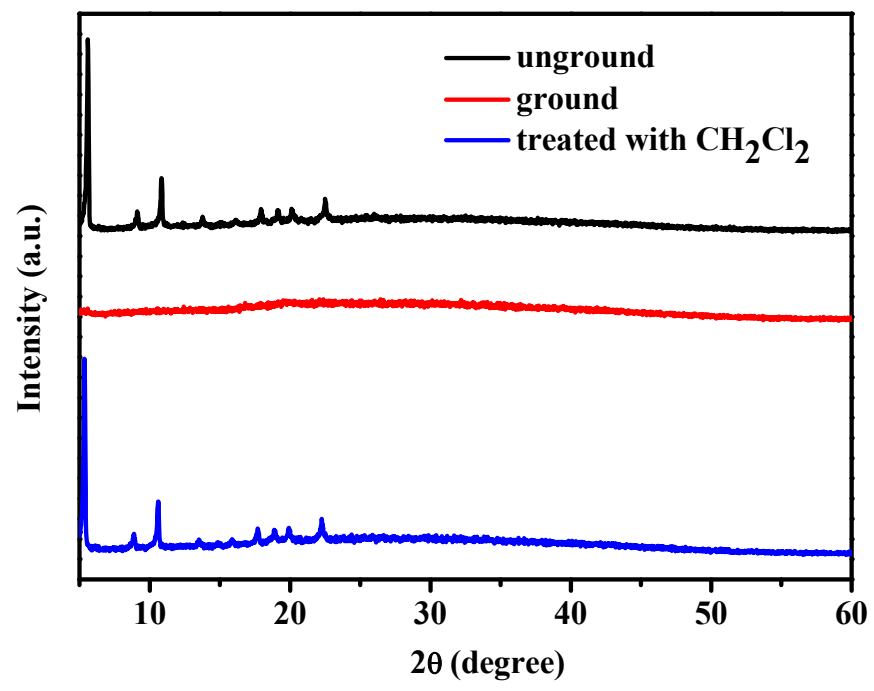

Figure S26. Powder XRD patterns of compound $\mathbf{6}$ in various solid states: the unground powder, the ground powder, the powder after treatment with dichloromethane vapor.

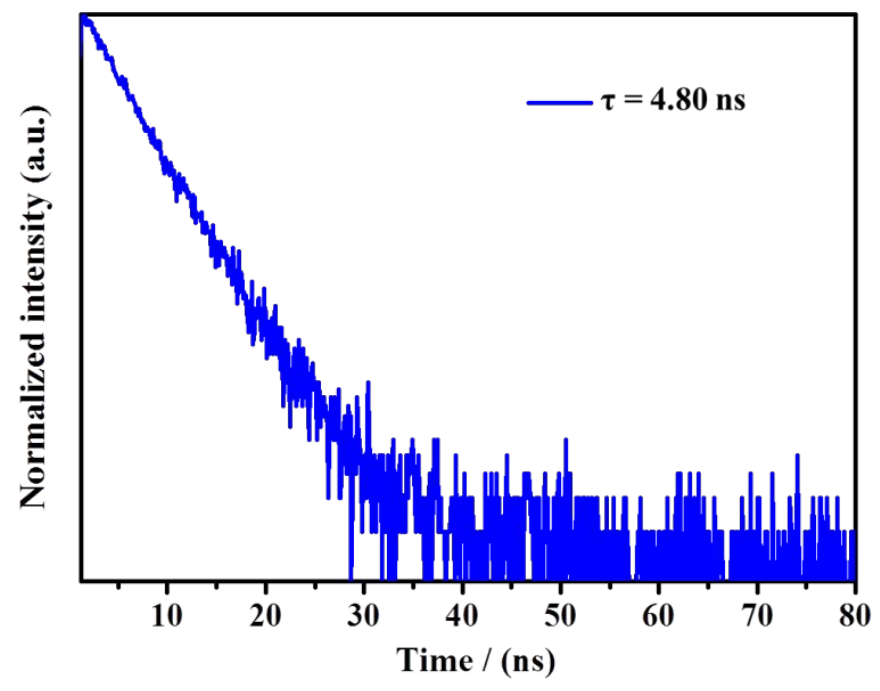

Figure S27. Decay curve (Emission wavelength: $490 \mathrm{~nm}$ ) of single crystals of 3. Excitation wavelength: $365 \mathrm{~nm}$. 


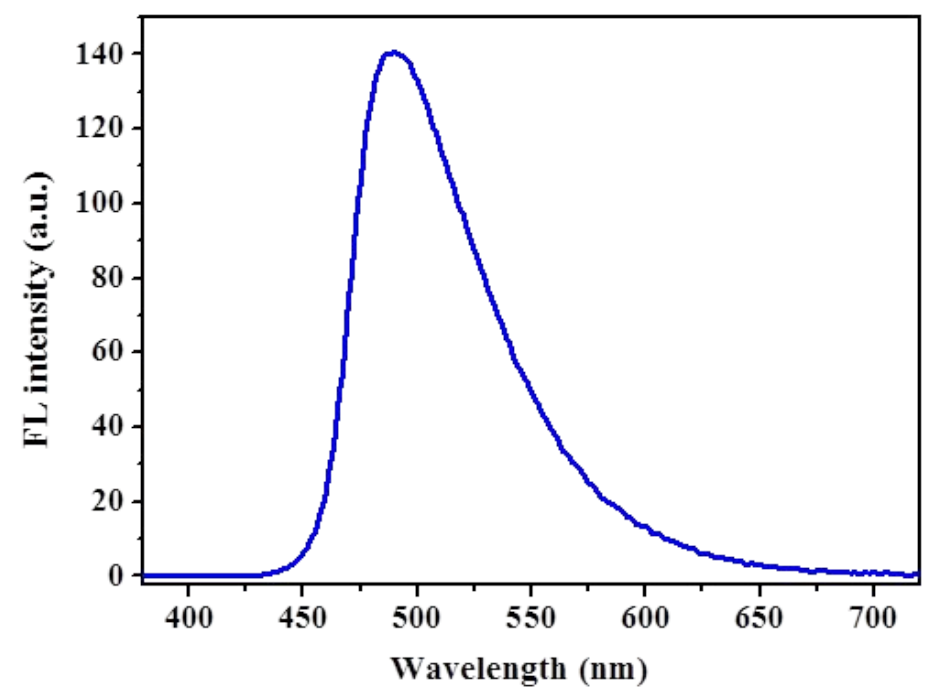

Figure S28. The fluorescence spectrum of single crystals of 3. Excitation wavelength: $365 \mathrm{~nm}$.

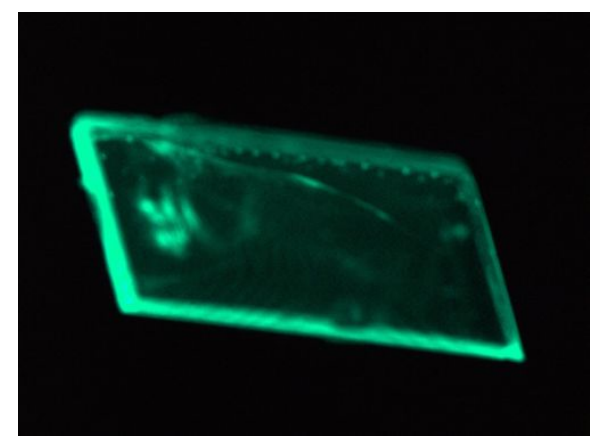

Figure S29. The fluorescence image of single crystals of 3. Excitation wavelength: $365 \mathrm{~nm}$.

\section{Table S1 and Table S2}

Table S1. Structure determination summary for the compound 3.

\begin{tabular}{cc}
\hline Empirical formula & $\mathrm{C}_{42} \mathrm{H}_{33} \mathrm{NO}_{2}$ \\
Formula weight & 583.69 \\
Temperature $(\mathrm{K})$ & $296(2)$ \\
Crystal system & Monoclinic \\
Space group & $\mathrm{P} 2(1) / \mathrm{c}$ \\
$a(\AA)$ & $9.5161(12)$ \\
$b(\AA)$ & $30.572(4)$ \\
$c(\AA)$ & $13.2584(12)$ \\
$\alpha(\mathrm{deg})$ & 90 \\
\hline
\end{tabular}




\begin{tabular}{|c|c|}
\hline$\beta(\mathrm{deg})$ & $125.371(6)$ \\
\hline$\gamma(\operatorname{deg})$ & 90 \\
\hline$V\left(\AA^{3}\right)$ & $3145.3(7)$ \\
\hline $\mathrm{Z}$ & 4 \\
\hline Absorption coefficient $\left(\mathrm{mm}^{-1}\right)$ & 0.075 \\
\hline $\mathrm{F}(000)$ & 1232 \\
\hline $\begin{array}{l}\text { Theta range for data collection } \\
\text { (deg) }\end{array}$ & 1.332 to 26.000 \\
\hline Index ranges & $-11<=\mathrm{h}<=11,-37<=\mathrm{k}<=27,-13<=\mathrm{k}<=16$ \\
\hline Reflections collected/unique & $\begin{array}{c}17032 / 6151 \\
\left(\mathrm{R}_{\text {int }}=0.1450\right)\end{array}$ \\
\hline Final $R$ indices $[\mathrm{I}>2 \operatorname{sigma}(\mathrm{I})]$ & $\mathrm{R}_{1}=0.0612, \mathrm{wR}_{2}=0.1173$ \\
\hline $\mathrm{R}$ indices (all data) & $\mathrm{R}_{1}=0.2165, \mathrm{wR}_{2}=0.1735$ \\
\hline Goodness-of-fit on $\mathrm{F}^{2}$ & 0.900 \\
\hline $\begin{array}{l}\text { Largest difference peak and } \\
\text { hole }\left(\mathrm{e} \AA^{-3}\right)\end{array}$ & $0.195,-0.214$ \\
\hline
\end{tabular}

Table S2. Bond lengths $[\AA]$ and angles $\left[^{\circ}\right]$ of $\mathbf{3}$.

$\begin{array}{lclc}\mathrm{N}(1)-\mathrm{C}(38) & 1.396(4) & \mathrm{C}(28)-\mathrm{H}(28 \mathrm{~A}) & 0.9300 \\ \mathrm{~N}(1)-\mathrm{C}(37) & 1.408(4) & \mathrm{C}(7)-\mathrm{C}(14) & 1.346(4) \\ \mathrm{N}(1)-\mathrm{C}(39) & 1.470(4) & \mathrm{C}(7)-\mathrm{C}(4) & 1.498(4) \\ \mathrm{O}(2)-\mathrm{C}(38) & 1.221(4) & \mathrm{C}(7)-\mathrm{C}(8) & 1.500(4) \\ \mathrm{C}(27)-\mathrm{C}(28) & 1.376(4) & \mathrm{C}(24)-\mathrm{C}(23) & 1.382(4) \\ \mathrm{C}(27)-\mathrm{C}(32) & 1.432(4) & \mathrm{C}(14)-\mathrm{C}(21) & 1.489(4) \\ \mathrm{C}(27)-\mathrm{C}(24) & 1.486(4) & \mathrm{C}(38)-\mathrm{C}(33) & 1.467(4) \\ \mathrm{C}(32)-\mathrm{C}(31) & 1.420(4) & \mathrm{C}(31)-\mathrm{C}(33) & 1.409(4) \\ \mathrm{C}(32)-\mathrm{C}(36) & 1.416(4) & \mathrm{C}(21)-\mathrm{C}(22) & 1.388(4) \\ \mathrm{C}(15)-\mathrm{C}(20) & 1.376(4) & \mathrm{C}(21)-\mathrm{C}(26) & 1.385(4) \\ \mathrm{C}(15)-\mathrm{C}(16) & 1.387(4) & \mathrm{C}(26)-\mathrm{H}(26 \mathrm{~A}) & 0.9300 \\ \mathrm{C}(15)-\mathrm{C}(14) & 1.501(4) & \mathrm{C}(8)-\mathrm{C}(9) & 1.388(4) \\ \mathrm{O}(1)-\mathrm{C}(37) & 1.219(3) & \mathrm{C}(8)-\mathrm{C}(13) & 1.385(4) \\ \mathrm{C}(30)-\mathrm{C}(29) & 1.374(4) & \mathrm{C}(33)-\mathrm{C}(34) & 1.364(4) \\ \mathrm{C}(30)-\mathrm{C}(31) & 1.407(4) & \mathrm{C}(23)-\mathrm{C}(22) & 1.375(4) \\ \mathrm{C}(30)-\mathrm{C}(37) & 1.472(4) & \mathrm{C}(23)-\mathrm{H}(23 \mathrm{~A}) & 0.9300 \\ \mathrm{C}(36)-\mathrm{C}(35) & 1.365(4) & \mathrm{C}(29)-\mathrm{H}(29 \mathrm{~A}) & 0.9300 \\ \mathrm{C}(36)-\mathrm{H}(36 \mathrm{~A}) & 0.9300 & \mathrm{C}(3)-\mathrm{C}(2) & 1.377(4) \\ \mathrm{C}(25)-\mathrm{C}(26) & 1.379(4) & \mathrm{C}(3)-\mathrm{C}(4) & 1.384(4) \\ \mathrm{C}(25)-\mathrm{C}(24) & 1.392(4) & \mathrm{C}(3)-\mathrm{H}(3 \mathrm{~A}) & 0.9300 \\ \mathrm{C}(25)-\mathrm{H}(25 \mathrm{~A}) & 0.9300 & \mathrm{C}(19)-\mathrm{C}(18) & 1.378(5) \\ \mathrm{C}(28)-\mathrm{C}(29) & 1.393(4) & \mathrm{C}(19)-\mathrm{C}(20) & 1.382(4) \\ & & & \end{array}$




\begin{tabular}{|c|c|c|c|}
\hline C(19)-H(19A) & 0.9300 & $\mathrm{C}\left(41^{\prime}\right)-\mathrm{H}(41 \mathrm{D})$ & 0.9700 \\
\hline$C(16)-C(17)$ & $1.376(4)$ & $\mathrm{C}\left(40^{\prime}\right)-\mathrm{H}(40 \mathrm{C})$ & 0.9700 \\
\hline $\mathrm{C}(16)-\mathrm{H}(16 \mathrm{~A})$ & 0.9300 & $\mathrm{C}\left(40^{\prime}\right)-\mathrm{H}(40 \mathrm{D})$ & $0.970 \mathrm{c}$ \\
\hline $\mathrm{C}(22)-\mathrm{H}(22 \mathrm{~A})$ & 0.9300 & $\mathrm{C}(38)-\mathrm{N}(1)-\mathrm{C}(37)$ & $123.5(3)$ \\
\hline$C(4)-C(5)$ & $1.375(4)$ & $\mathrm{C}(38)-\mathrm{N}(1)-\mathrm{C}(39)$ & $119.4(3)$ \\
\hline $\mathrm{C}(20)-\mathrm{H}(20 \mathrm{~A})$ & 0.9300 & C(37)-N(1)-C(39) & $117.0(3)$ \\
\hline$C(5)-C(6)$ & $1.382(5)$ & $C(28)-C(27)-C(32)$ & $118.4(3)$ \\
\hline $\mathrm{C}(5)-\mathrm{H}(5 \mathrm{~A})$ & 0.9300 & $C(28)-C(27)-C(24)$ & $121.8(3)$ \\
\hline$C(35)-C(34)$ & $1.389(4)$ & $\mathrm{C}(32)-\mathrm{C}(27)-\mathrm{C}(24)$ & $119.8(3)$ \\
\hline $\mathrm{C}(35)-\mathrm{H}(35 \mathrm{~A})$ & 0.9300 & $\mathrm{C}(31)-\mathrm{C}(32)-\mathrm{C}(36)$ & $118.0(3)$ \\
\hline $\mathrm{C}(39)-\mathrm{C}\left(40^{\prime}\right)$ & $1.49(2)$ & $\mathrm{C}(31)-\mathrm{C}(32)-\mathrm{C}(27)$ & 119.1(3) \\
\hline$C(39)-C(40)$ & $1.569(14)$ & $C(36)-C(32)-C(27)$ & $122.9(3)$ \\
\hline C(39)-H(39A) & 0.9700 & $C(20)-C(15)-C(16)$ & $118.4(3)$ \\
\hline C(39)-H(39B) & 0.9700 & $C(20)-C(15)-C(14)$ & 119.6(3) \\
\hline$C(12)-C(11)$ & $1.361(5)$ & $C(16)-C(15)-C(14)$ & $122.0(3)$ \\
\hline $\mathrm{C}(12)-\mathrm{C}(13)$ & $1.380(4)$ & $\mathrm{C}(29)-\mathrm{C}(30)-\mathrm{C}(31)$ & $119.7(3)$ \\
\hline $\mathrm{C}(12)-\mathrm{H}(12 \mathrm{~A})$ & 0.9300 & $C(29)-C(30)-C(37)$ & $119.7(3)$ \\
\hline $\mathrm{C}(34)-\mathrm{H}(34 \mathrm{~A})$ & 0.9300 & $C(31)-C(30)-C(37)$ & $120.6(3)$ \\
\hline $\mathrm{C}(18)-\mathrm{C}(17)$ & $1.368(5)$ & $C(35)-C(36)-C(32)$ & $120.9(3)$ \\
\hline $\mathrm{C}(18)-\mathrm{H}(18 \mathrm{~A})$ & 0.9300 & $C(35)-C(36)-H(36 A)$ & 119.6 \\
\hline $\mathrm{C}(17)-\mathrm{H}(17 \mathrm{~A})$ & 0.9300 & $\mathrm{C}(32)-\mathrm{C}(36)-\mathrm{H}(36 \mathrm{~A})$ & 119.6 \\
\hline $\mathrm{C}(9)-\mathrm{C}(10)$ & $1.386(4)$ & $C(26)-C(25)-C(24)$ & $120.6(3)$ \\
\hline $\mathrm{C}(9)-\mathrm{H}(9 \mathrm{~A})$ & 0.9300 & $\mathrm{C}(26)-\mathrm{C}(25)-\mathrm{H}(25 \mathrm{~A})$ & 119.7 \\
\hline $\mathrm{C}(1)-\mathrm{C}(2)$ & $1.357(6)$ & $\mathrm{C}(24)-\mathrm{C}(25)-\mathrm{H}(25 \mathrm{~A})$ & 119.7 \\
\hline$C(1)-C(6)$ & $1.363(5)$ & $\mathrm{C}(27)-\mathrm{C}(28)-\mathrm{C}(29)$ & $122.2(3)$ \\
\hline $\mathrm{C}(1)-\mathrm{H}(1 \mathrm{~A})$ & 0.9300 & $\mathrm{C}(27)-\mathrm{C}(28)-\mathrm{H}(28 \mathrm{~A})$ & 118.9 \\
\hline $\mathrm{C}(13)-\mathrm{H}(13 \mathrm{~A})$ & 0.9300 & $\mathrm{C}(29)-\mathrm{C}(28)-\mathrm{H}(28 \mathrm{~A})$ & 118.9 \\
\hline $\mathrm{C}(2)-\mathrm{H}(2 \mathrm{~A})$ & 0.9300 & $C(14)-C(7)-C(4)$ & $120.5(3)$ \\
\hline $\mathrm{C}(11)-\mathrm{C}(10)$ & $1.371(5)$ & $\mathrm{C}(14)-\mathrm{C}(7)-\mathrm{C}(8)$ & $124.4(3)$ \\
\hline $\mathrm{C}(11)-\mathrm{H}(11 \mathrm{~A})$ & 0.9300 & $C(4)-C(7)-C(8)$ & $115.1(2)$ \\
\hline $\mathrm{C}(6)-\mathrm{H}(6 \mathrm{~A})$ & 0.9300 & $C(23)-C(24)-C(25)$ & $118.4(3)$ \\
\hline $\mathrm{C}(10)-\mathrm{H}(10 \mathrm{~A})$ & 0.9300 & $\mathrm{C}(23)-\mathrm{C}(24)-\mathrm{C}(27)$ & $122.0(3)$ \\
\hline $\mathrm{C}(42)-\mathrm{C}\left(41^{\prime}\right)$ & $1.555(17)$ & $C(25)-C(24)-C(27)$ & $119.6(3)$ \\
\hline $\mathrm{C}(42)-\mathrm{C}(41)$ & $1.597(14)$ & $C(7)-C(14)-C(21)$ & $122.8(3)$ \\
\hline $\mathrm{C}(42)-\mathrm{H}(42 \mathrm{~A})$ & 0.9600 & $C(7)-C(14)-C(15)$ & $122.5(3)$ \\
\hline $\mathrm{C}(42)-\mathrm{H}(42 \mathrm{~B})$ & 0.9600 & $C(21)-C(14)-C(15)$ & $114.7(2)$ \\
\hline $\mathrm{C}(42)-\mathrm{H}(42 \mathrm{C})$ & 0.9600 & $\mathrm{O}(2)-\mathrm{C}(38)-\mathrm{N}(1)$ & $119.9(3)$ \\
\hline$C(40)-C(41)$ & $1.45(2)$ & $\mathrm{O}(2)-\mathrm{C}(38)-\mathrm{C}(33)$ & $122.1(3)$ \\
\hline $\mathrm{C}(40)-\mathrm{H}(40 \mathrm{~A})$ & 0.9700 & $\mathrm{~N}(1)-\mathrm{C}(38)-\mathrm{C}(33)$ & $118.0(3)$ \\
\hline $\mathrm{C}(40)-\mathrm{H}(40 \mathrm{~B})$ & 0.9700 & $\mathrm{C}(30)-\mathrm{C}(31)-\mathrm{C}(33)$ & $120.2(3)$ \\
\hline $\mathrm{C}(41)-\mathrm{H}(41 \mathrm{~A})$ & 0.9700 & $C(30)-C(31)-C(32)$ & $120.2(3)$ \\
\hline $\mathrm{C}(41)-\mathrm{H}(41 \mathrm{~B})$ & 0.9700 & $\mathrm{C}(33)-\mathrm{C}(31)-\mathrm{C}(32)$ & $119.7(3)$ \\
\hline$C\left(41^{\prime}\right)-C\left(40^{\prime}\right)$ & $1.54(3)$ & $C(22)-C(21)-C(26)$ & $117.6(3)$ \\
\hline $\mathrm{C}\left(41^{\prime}\right)-\mathrm{H}(41 \mathrm{C})$ & 0.9700 & $C(22)-C(21)-C(14)$ & $120.9(3)$ \\
\hline
\end{tabular}




\begin{tabular}{|c|c|c|c|}
\hline$C(26)-C(21)-C(14)$ & $121.5(3)$ & $\mathrm{N}(1)-\mathrm{C}(39)-\mathrm{C}(40)$ & $113.3(5)$ \\
\hline $\mathrm{C}(25)-\mathrm{C}(26)-\mathrm{C}(21)$ & $121.1(3)$ & $\mathrm{N}(1)-\mathrm{C}(39)-\mathrm{H}(39 \mathrm{~A})$ & 108.9 \\
\hline $\mathrm{C}(25)-\mathrm{C}(26)-\mathrm{H}(26 \mathrm{~A})$ & 119.4 & $\mathrm{C}(40)-\mathrm{C}(39)-\mathrm{H}(39 \mathrm{~A})$ & 108.9 \\
\hline $\mathrm{C}(21)-\mathrm{C}(26)-\mathrm{H}(26 \mathrm{~A})$ & 119.4 & N(1)-C(39)-H(39B) & 108.9 \\
\hline $\mathrm{C}(9)-\mathrm{C}(8)-\mathrm{C}(13)$ & $117.3(3)$ & $\mathrm{C}(40)-\mathrm{C}(39)-\mathrm{H}(39 \mathrm{~B})$ & 108.9 \\
\hline $\mathrm{C}(9)-\mathrm{C}(8)-\mathrm{C}(7)$ & $122.6(3)$ & $\mathrm{H}(39 \mathrm{~A})-\mathrm{C}(39)-\mathrm{H}(39 \mathrm{~B})$ & 107.7 \\
\hline $\mathrm{C}(13)-\mathrm{C}(8)-\mathrm{C}(7)$ & $120.1(3)$ & $\mathrm{C}(11)-\mathrm{C}(12)-\mathrm{C}(13)$ & $120.2(3)$ \\
\hline $\mathrm{C}(34)-\mathrm{C}(33)-\mathrm{C}(31)$ & $120.0(3)$ & $\mathrm{C}(11)-\mathrm{C}(12)-\mathrm{H}(12 \mathrm{~A})$ & 119.9 \\
\hline $\mathrm{C}(34)-\mathrm{C}(33)-\mathrm{C}(38)$ & $119.7(3)$ & $\mathrm{C}(13)-\mathrm{C}(12)-\mathrm{H}(12 \mathrm{~A})$ & 119.9 \\
\hline $\mathrm{C}(31)-\mathrm{C}(33)-\mathrm{C}(38)$ & $120.3(3)$ & C(33)-C(34)-C(35) & $121.0(3)$ \\
\hline$C(22)-C(23)-C(24)$ & $120.5(3)$ & $\mathrm{C}(33)-\mathrm{C}(34)-\mathrm{H}(34 \mathrm{~A})$ & 119.5 \\
\hline $\mathrm{C}(22)-\mathrm{C}(23)-\mathrm{H}(23 \mathrm{~A})$ & 119.8 & $\mathrm{C}(35)-\mathrm{C}(34)-\mathrm{H}(34 \mathrm{~A})$ & 119.5 \\
\hline $\mathrm{C}(24)-\mathrm{C}(23)-\mathrm{H}(23 \mathrm{~A})$ & 119.8 & $\mathrm{C}(17)-\mathrm{C}(18)-\mathrm{C}(19)$ & $120.2(3)$ \\
\hline $\mathrm{C}(30)-\mathrm{C}(29)-\mathrm{C}(28)$ & $120.4(3)$ & $\mathrm{C}(17)-\mathrm{C}(18)-\mathrm{H}(18 \mathrm{~A})$ & 119.9 \\
\hline $\mathrm{C}(30)-\mathrm{C}(29)-\mathrm{H}(29 \mathrm{~A})$ & 119.8 & $\mathrm{C}(19)-\mathrm{C}(18)-\mathrm{H}(18 \mathrm{~A})$ & 119.9 \\
\hline $\mathrm{C}(28)-\mathrm{C}(29)-\mathrm{H}(29 \mathrm{~A})$ & 119.8 & $\mathrm{C}(18)-\mathrm{C}(17)-\mathrm{C}(16)$ & $120.1(4)$ \\
\hline $\mathrm{C}(2)-\mathrm{C}(3)-\mathrm{C}(4)$ & $120.8(4)$ & $\mathrm{C}(18)-\mathrm{C}(17)-\mathrm{H}(17 \mathrm{~A})$ & 119.9 \\
\hline $\mathrm{C}(2)-\mathrm{C}(3)-\mathrm{H}(3 \mathrm{~A})$ & 119.6 & $\mathrm{C}(16)-\mathrm{C}(17)-\mathrm{H}(17 \mathrm{~A})$ & 119.9 \\
\hline $\mathrm{C}(4)-\mathrm{C}(3)-\mathrm{H}(3 \mathrm{~A})$ & 119.6 & $\mathrm{C}(10)-\mathrm{C}(9)-\mathrm{C}(8)$ & $120.9(3)$ \\
\hline$C(18)-C(19)-C(20)$ & 119.3(3) & $\mathrm{C}(10)-\mathrm{C}(9)-\mathrm{H}(9 \mathrm{~A})$ & 119.6 \\
\hline $\mathrm{C}(18)-\mathrm{C}(19)-\mathrm{H}(19 \mathrm{~A})$ & 120.3 & $\mathrm{C}(8)-\mathrm{C}(9)-\mathrm{H}(9 \mathrm{~A})$ & 119.6 \\
\hline $\mathrm{C}(20)-\mathrm{C}(19)-\mathrm{H}(19 \mathrm{~A})$ & 120.3 & $C(2)-C(1)-C(6)$ & $119.4(4)$ \\
\hline $\mathrm{C}(17)-\mathrm{C}(16)-\mathrm{C}(15)$ & $120.7(3)$ & $\mathrm{C}(2)-\mathrm{C}(1)-\mathrm{H}(1 \mathrm{~A})$ & 120.3 \\
\hline $\mathrm{C}(17)-\mathrm{C}(16)-\mathrm{H}(16 \mathrm{~A})$ & 119.6 & $\mathrm{C}(6)-\mathrm{C}(1)-\mathrm{H}(1 \mathrm{~A})$ & 120.3 \\
\hline $\mathrm{C}(15)-\mathrm{C}(16)-\mathrm{H}(16 \mathrm{~A})$ & 119.6 & $\mathrm{C}(12)-\mathrm{C}(13)-\mathrm{C}(8)$ & $121.6(3)$ \\
\hline $\mathrm{O}(1)-\mathrm{C}(37)-\mathrm{N}(1)$ & $120.0(3)$ & $\mathrm{C}(12)-\mathrm{C}(13)-\mathrm{H}(13 \mathrm{~A})$ & 119.2 \\
\hline $\mathrm{O}(1)-\mathrm{C}(37)-\mathrm{C}(30)$ & $122.8(3)$ & $\mathrm{C}(8)-\mathrm{C}(13)-\mathrm{H}(13 \mathrm{~A})$ & 119.2 \\
\hline $\mathrm{N}(1)-\mathrm{C}(37)-\mathrm{C}(30)$ & $117.2(3)$ & $\mathrm{C}(1)-\mathrm{C}(2)-\mathrm{C}(3)$ & $120.7(4)$ \\
\hline $\mathrm{C}(23)-\mathrm{C}(22)-\mathrm{C}(21)$ & $121.6(3)$ & $\mathrm{C}(1)-\mathrm{C}(2)-\mathrm{H}(2 \mathrm{~A})$ & 119.6 \\
\hline $\mathrm{C}(23)-\mathrm{C}(22)-\mathrm{H}(22 \mathrm{~A})$ & 119.2 & $\mathrm{C}(3)-\mathrm{C}(2)-\mathrm{H}(2 \mathrm{~A})$ & 119.6 \\
\hline $\mathrm{C}(21)-\mathrm{C}(22)-\mathrm{H}(22 \mathrm{~A})$ & 119.2 & $\mathrm{C}(12)-\mathrm{C}(11)-\mathrm{C}(10)$ & $119.7(3)$ \\
\hline$C(5)-C(4)-C(3)$ & $117.5(3)$ & $\mathrm{C}(12)-\mathrm{C}(11)-\mathrm{H}(11 \mathrm{~A})$ & 120.2 \\
\hline$C(5)-C(4)-C(7)$ & $122.1(3)$ & $\mathrm{C}(10)-\mathrm{C}(11)-\mathrm{H}(11 \mathrm{~A})$ & 120.2 \\
\hline $\mathrm{C}(3)-\mathrm{C}(4)-\mathrm{C}(7)$ & $120.3(3)$ & $\mathrm{C}(1)-\mathrm{C}(6)-\mathrm{C}(5)$ & $120.2(4)$ \\
\hline$C(15)-C(20)-C(19)$ & $121.2(3)$ & $\mathrm{C}(1)-\mathrm{C}(6)-\mathrm{H}(6 \mathrm{~A})$ & 119.9 \\
\hline$C(15)-C(20)-H(20 A)$ & 119.4 & $\mathrm{C}(5)-\mathrm{C}(6)-\mathrm{H}(6 \mathrm{~A})$ & 119.9 \\
\hline $\mathrm{C}(19)-\mathrm{C}(20)-\mathrm{H}(20 \mathrm{~A})$ & 119.4 & $C(11)-C(10)-C(9)$ & $120.4(3)$ \\
\hline$C(4)-C(5)-C(6)$ & $121.2(4)$ & $\mathrm{C}(11)-\mathrm{C}(10)-\mathrm{H}(10 \mathrm{~A})$ & 119.8 \\
\hline $\mathrm{C}(4)-\mathrm{C}(5)-\mathrm{H}(5 \mathrm{~A})$ & 119.4 & $\mathrm{C}(9)-\mathrm{C}(10)-\mathrm{H}(10 \mathrm{~A})$ & 119.8 \\
\hline $\mathrm{C}(6)-\mathrm{C}(5)-\mathrm{H}(5 \mathrm{~A})$ & 119.4 & $\mathrm{C}(41)-\mathrm{C}(42)-\mathrm{H}(42 \mathrm{~A})$ & 109.5 \\
\hline $\mathrm{C}(36)-\mathrm{C}(35)-\mathrm{C}(34)$ & $120.4(3)$ & $\mathrm{C}(41)-\mathrm{C}(42)-\mathrm{H}(42 \mathrm{~B})$ & 109.5 \\
\hline $\mathrm{C}(36)-\mathrm{C}(35)-\mathrm{H}(35 \mathrm{~A})$ & 119.8 & $\mathrm{H}(42 \mathrm{~A})-\mathrm{C}(42)-\mathrm{H}(42 \mathrm{~B})$ & 109.5 \\
\hline $\mathrm{C}(34)-\mathrm{C}(35)-\mathrm{H}(35 \mathrm{~A})$ & 119.8 & $\mathrm{C}(41)-\mathrm{C}(42)-\mathrm{H}(42 \mathrm{C})$ & 109.5 \\
\hline N(1)-C(39)-C(40') & $111.4(7)$ & $\mathrm{H}(42 \mathrm{~A})-\mathrm{C}(42)-\mathrm{H}(42 \mathrm{C})$ & 109.5 \\
\hline
\end{tabular}




$\begin{array}{lclc}\mathrm{H}(42 \mathrm{~B})-\mathrm{C}(42)-\mathrm{H}(42 \mathrm{C}) & 109.5 & \mathrm{C}\left(40^{\prime}\right)-\mathrm{C}\left(41^{\prime}\right)-\mathrm{C}(42) & 109.2(18) \\ \mathrm{C}(41)-\mathrm{C}(40)-\mathrm{C}(39) & 110.2(12) & \mathrm{C}\left(40^{\prime}\right)-\mathrm{C}\left(41^{\prime}\right)-\mathrm{H}(41 \mathrm{C}) & 109.8 \\ \mathrm{C}(41)-\mathrm{C}(40)-\mathrm{H}(40 \mathrm{~A}) & 109.6 & \mathrm{C}(42)-\mathrm{C}\left(41^{\prime}\right)-\mathrm{H}(41 \mathrm{C}) & 109.8 \\ \mathrm{C}(39)-\mathrm{C}(40)-\mathrm{H}(40 \mathrm{~A}) & 109.6 & \mathrm{C}\left(40^{\prime}\right)-\mathrm{C}\left(41^{\prime}\right)-\mathrm{H}(41 \mathrm{D}) & 109.8 \\ \mathrm{C}(41)-\mathrm{C}(40)-\mathrm{H}(40 \mathrm{~B}) & 109.6 & \mathrm{C}(42)-\mathrm{C}\left(41^{\prime}\right)-\mathrm{H}(41 \mathrm{D}) & 109.8 \\ \mathrm{C}(39)-\mathrm{C}(40)-\mathrm{H}(40 \mathrm{~B}) & 109.6 & \mathrm{H}(41 \mathrm{C})-\mathrm{C}\left(41^{\prime}\right)-\mathrm{H}(41 \mathrm{D}) & 108.3 \\ \mathrm{H}(40 \mathrm{~A})-\mathrm{C}(40)-\mathrm{H}(40 \mathrm{~B}) & 108.1 & \mathrm{C}\left(41^{\prime}\right)-\mathrm{C}\left(40^{\prime}\right)-\mathrm{C}(39) & 113.4(19) \\ \mathrm{C}(40)-\mathrm{C}(41)-\mathrm{C}(42) & 107.0(12) & \mathrm{C}\left(41^{\prime}\right)-\mathrm{C}\left(40^{\prime}\right)-\mathrm{H}(40 \mathrm{C}) & 108.9 \\ \mathrm{C}(40)-\mathrm{C}(41)-\mathrm{H}(41 \mathrm{~A}) & 110.3 & \mathrm{C}(39)-\mathrm{C}\left(40^{\prime}\right)-\mathrm{H}(40 \mathrm{C}) & 108.9 \\ \mathrm{C}(42)-\mathrm{C}(41)-\mathrm{H}(41 \mathrm{~A}) & 110.3 & \mathrm{C}\left(41^{\prime}\right)-\mathrm{C}\left(40^{\prime}\right)-\mathrm{H}(40 \mathrm{D}) & 108.9 \\ \mathrm{C}(40)-\mathrm{C}(41)-\mathrm{H}(41 \mathrm{~B}) & 110.3 & \mathrm{C}(39)-\mathrm{C}\left(40^{\prime}\right)-\mathrm{H}(40 \mathrm{D}) & 108.9 \\ \mathrm{C}(42)-\mathrm{C}(41)-\mathrm{H}(41 \mathrm{~B}) & 110.3 & \mathrm{H}(40 \mathrm{C})-\mathrm{C}\left(40^{\prime}\right)-\mathrm{H}(40 \mathrm{D}) & 107.7 \\ \mathrm{H}(41 \mathrm{~A})-\mathrm{C}(41)-\mathrm{H}(41 \mathrm{~B}) & 108.6 & & \\ \end{array}$

\section{Copies of NMR spectra and Mass spectra}

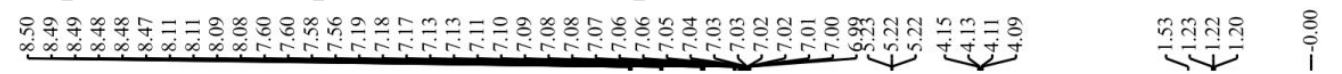
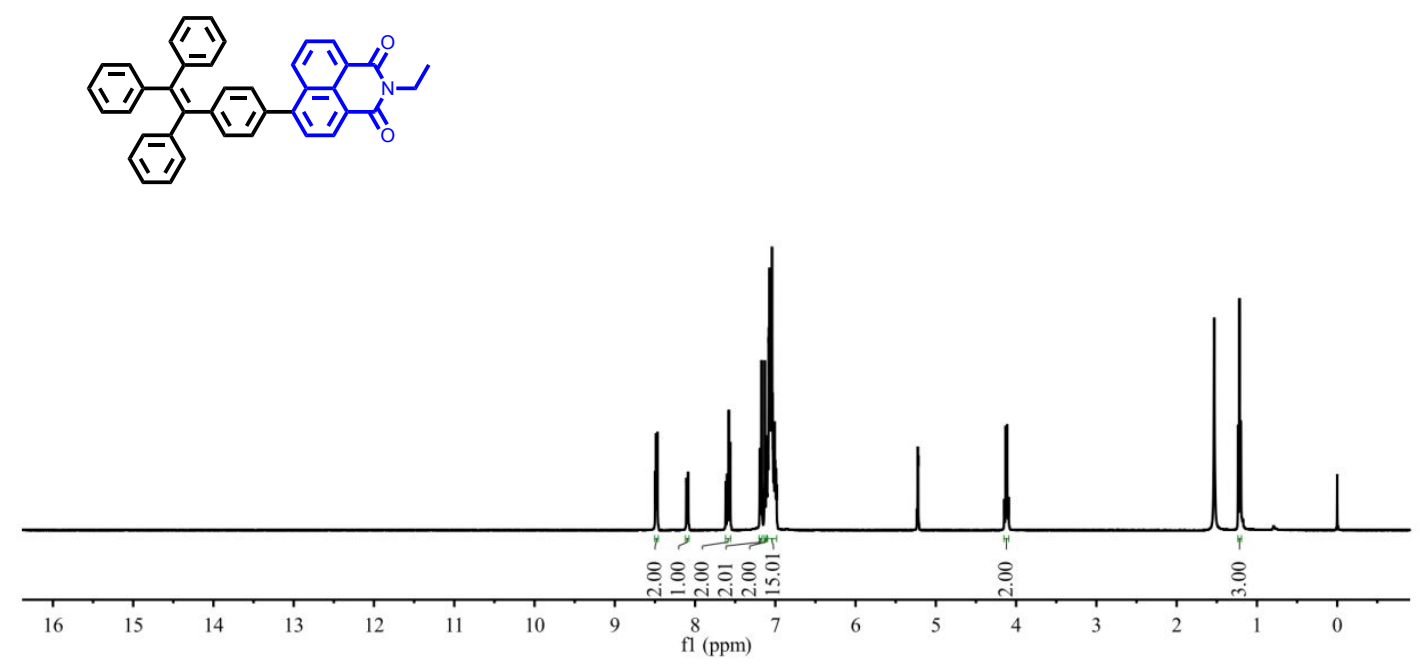

Figure S30. ${ }^{1} \mathrm{H}$ NMR spectrum of compound 1 . 
<smiles>CCN1C(=O)c2cccc3c(-c4ccc(C(=C(c5ccccc5)c5ccccc5)c5ccccc5)cc4)ccc(c23)C1=O</smiles>

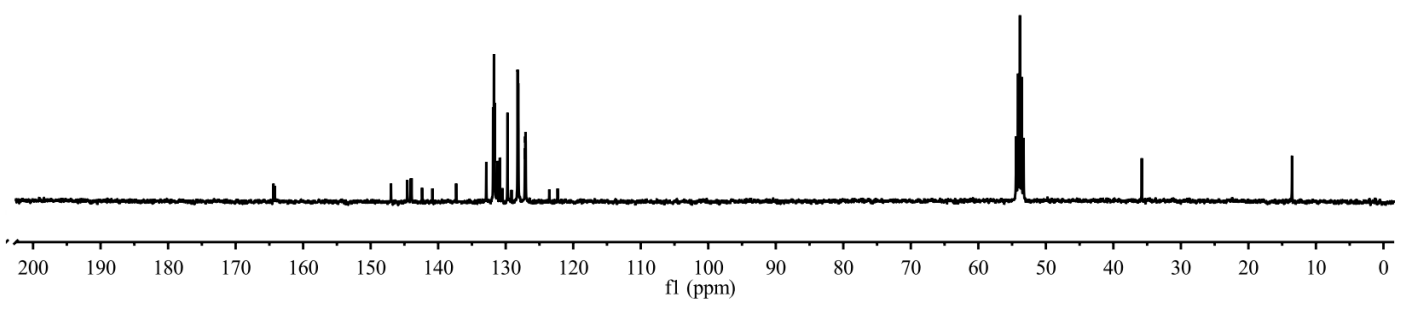

Figure S31. ${ }^{13} \mathrm{C}$ NMR spectrum of compound $\mathbf{1}$.

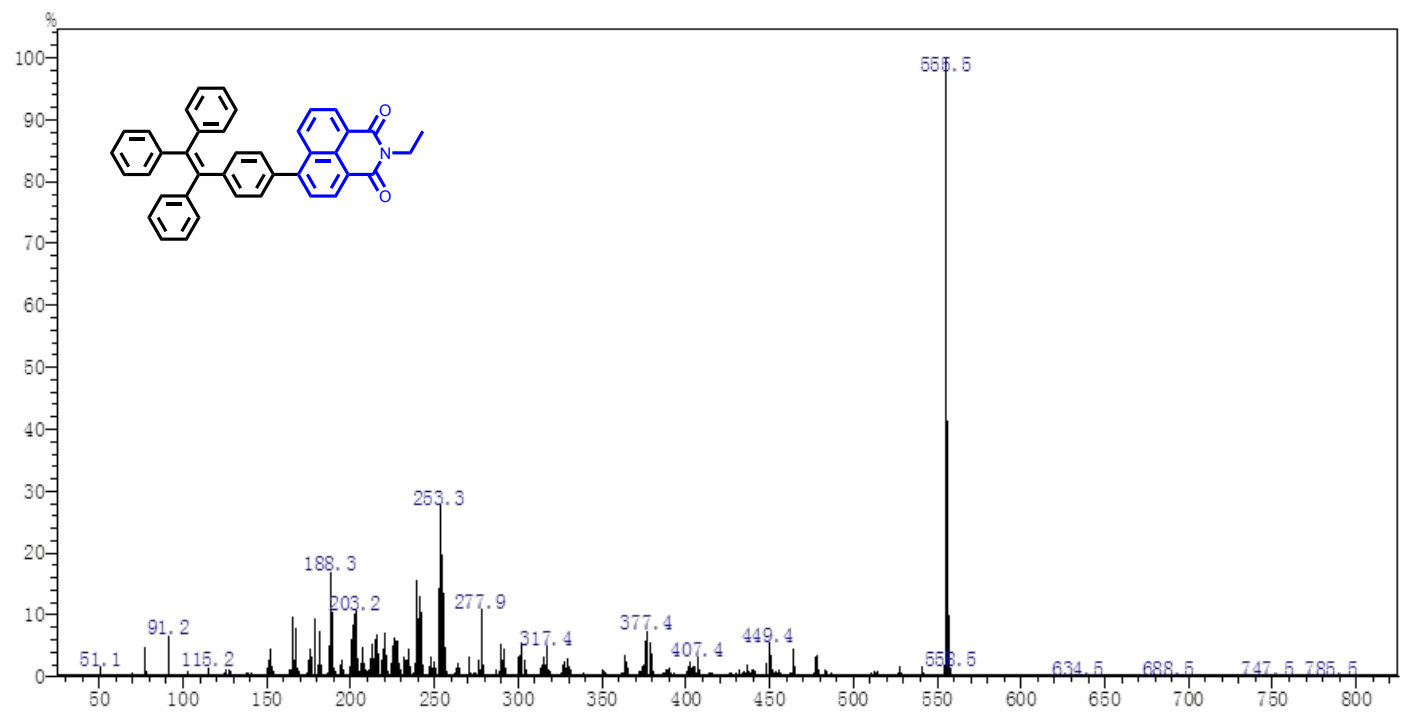

Figure S32. Mass spectrum of compound $\mathbf{1 .}$ 


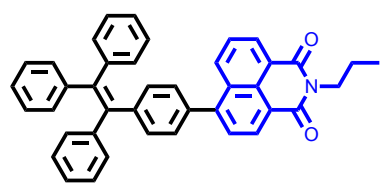

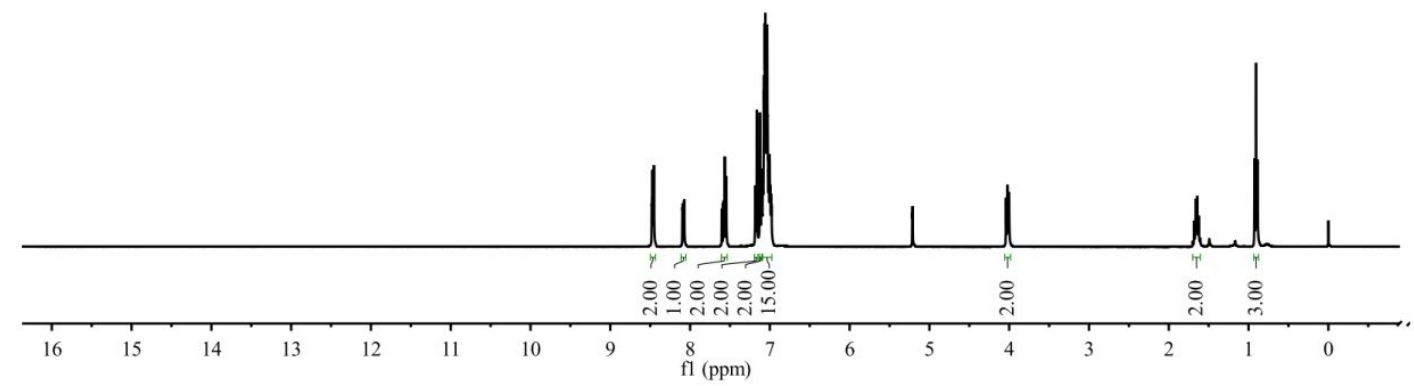

Figure S33. ${ }^{1} \mathrm{H}$ NMR spectrum of compound 2.

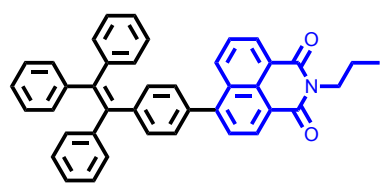

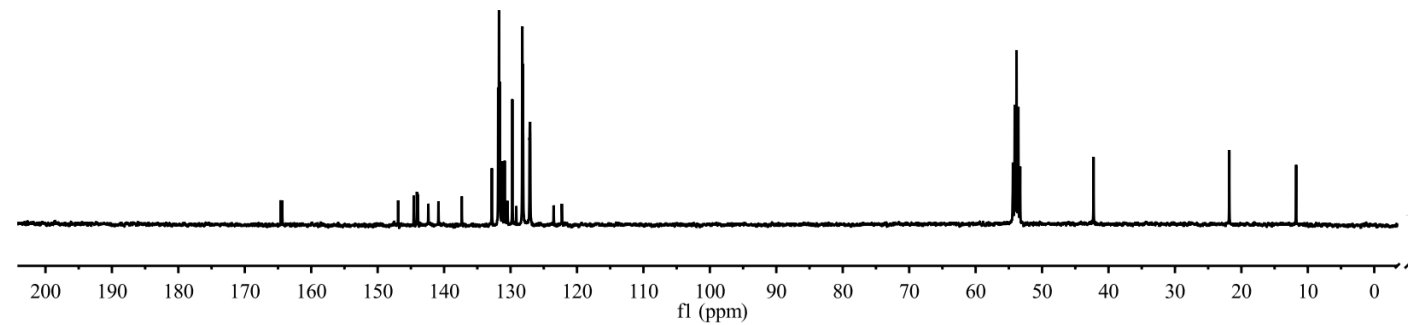

Figure S34. ${ }^{13} \mathrm{C}$ NMR spectrum of compound 2 . 


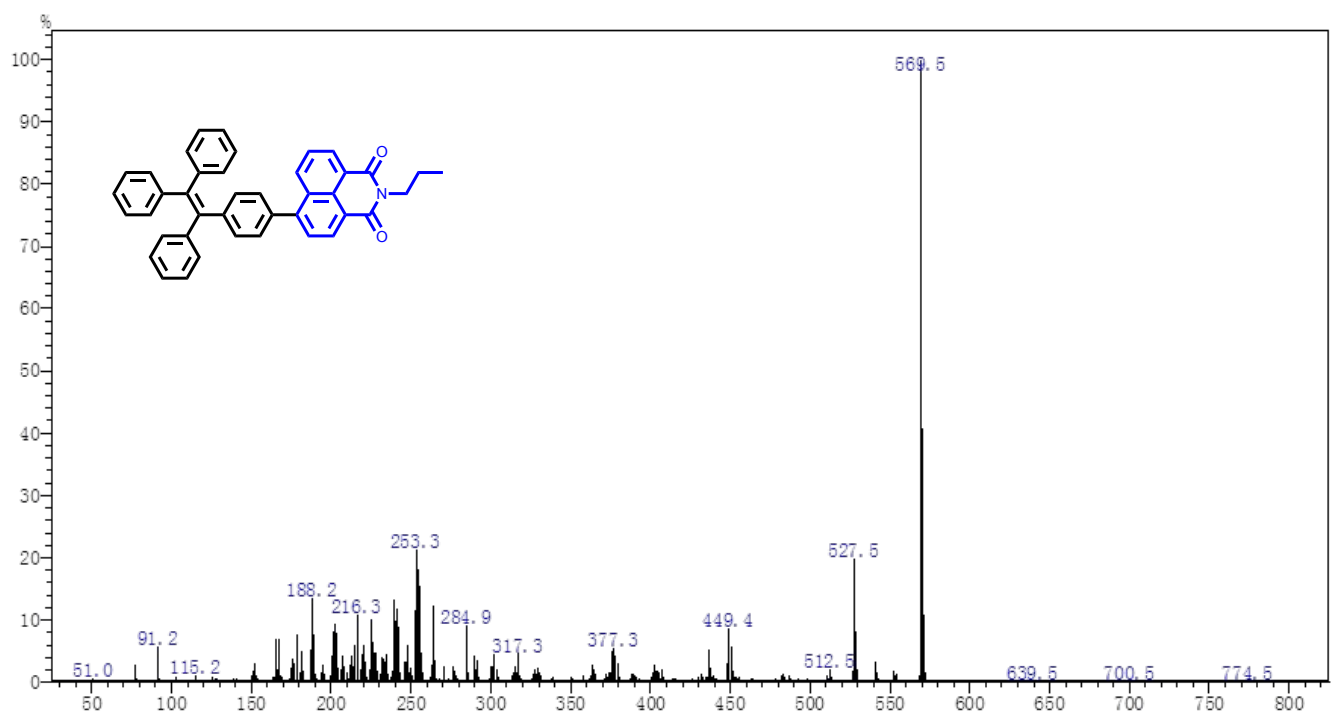

Figure S35. Mass spectrum of compound 2.

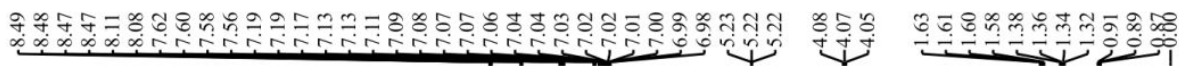

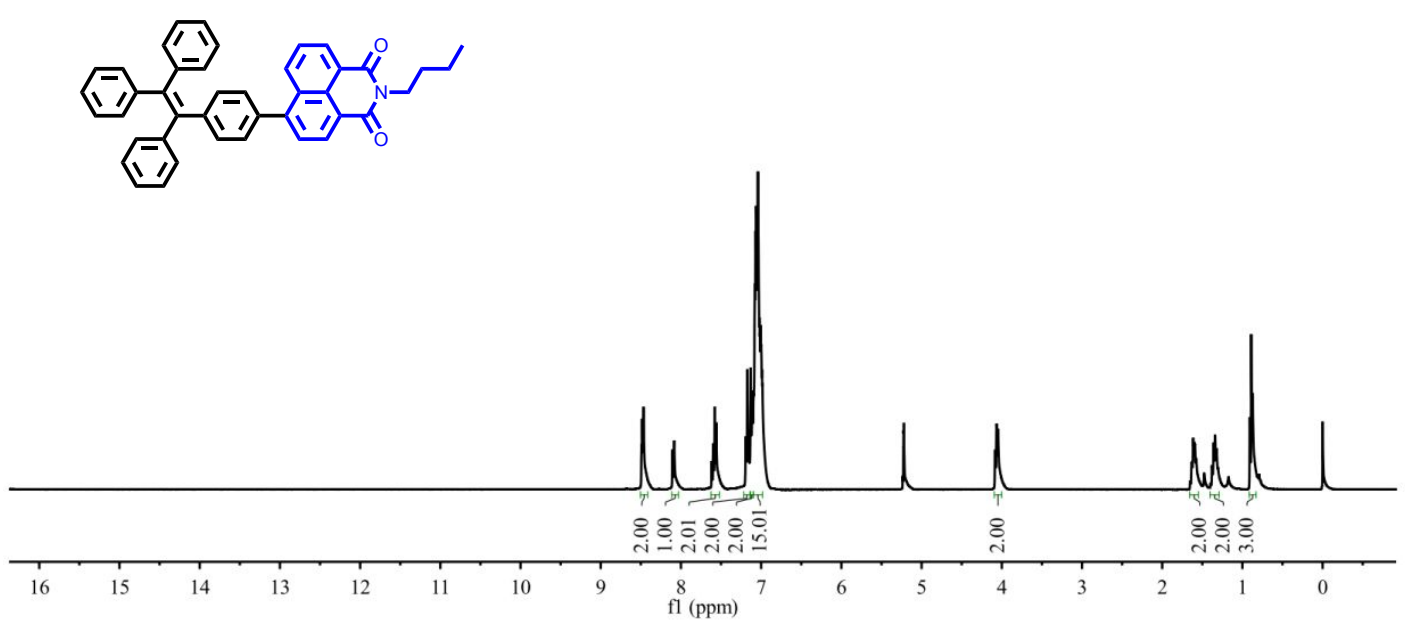

Figure S36. ${ }^{1} \mathrm{H}$ NMR spectrum of compound 3 . 

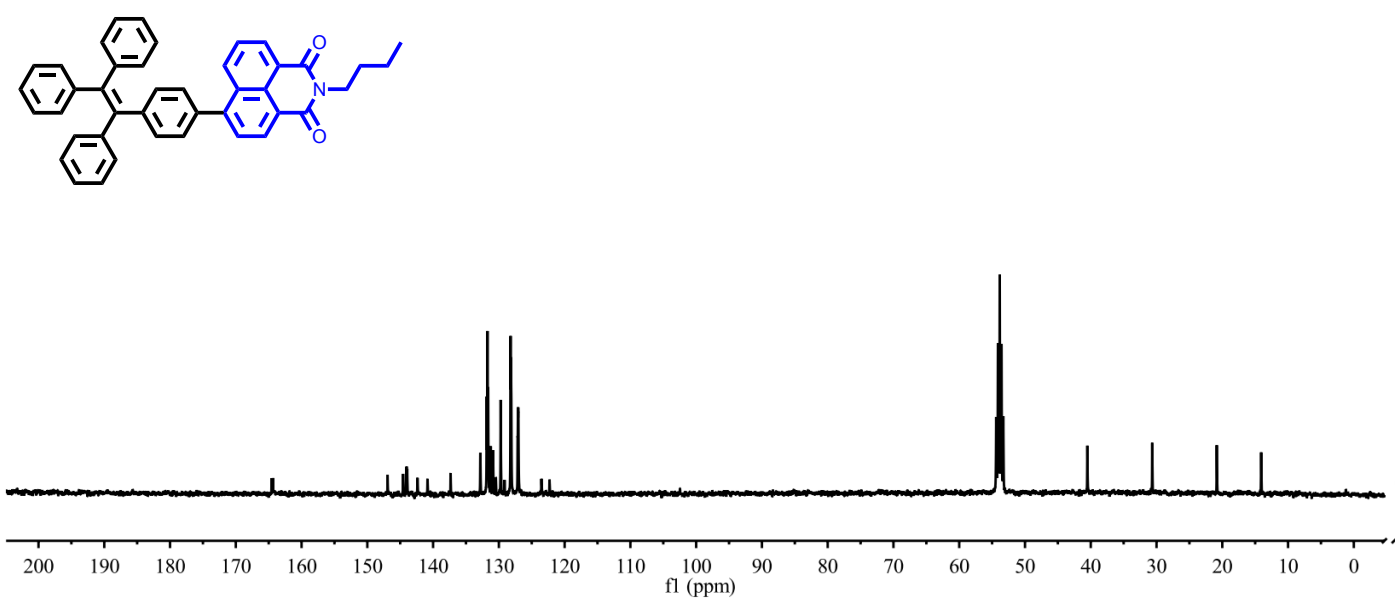

Figure S37. ${ }^{13} \mathrm{C}$ NMR spectrum of compound 3 .

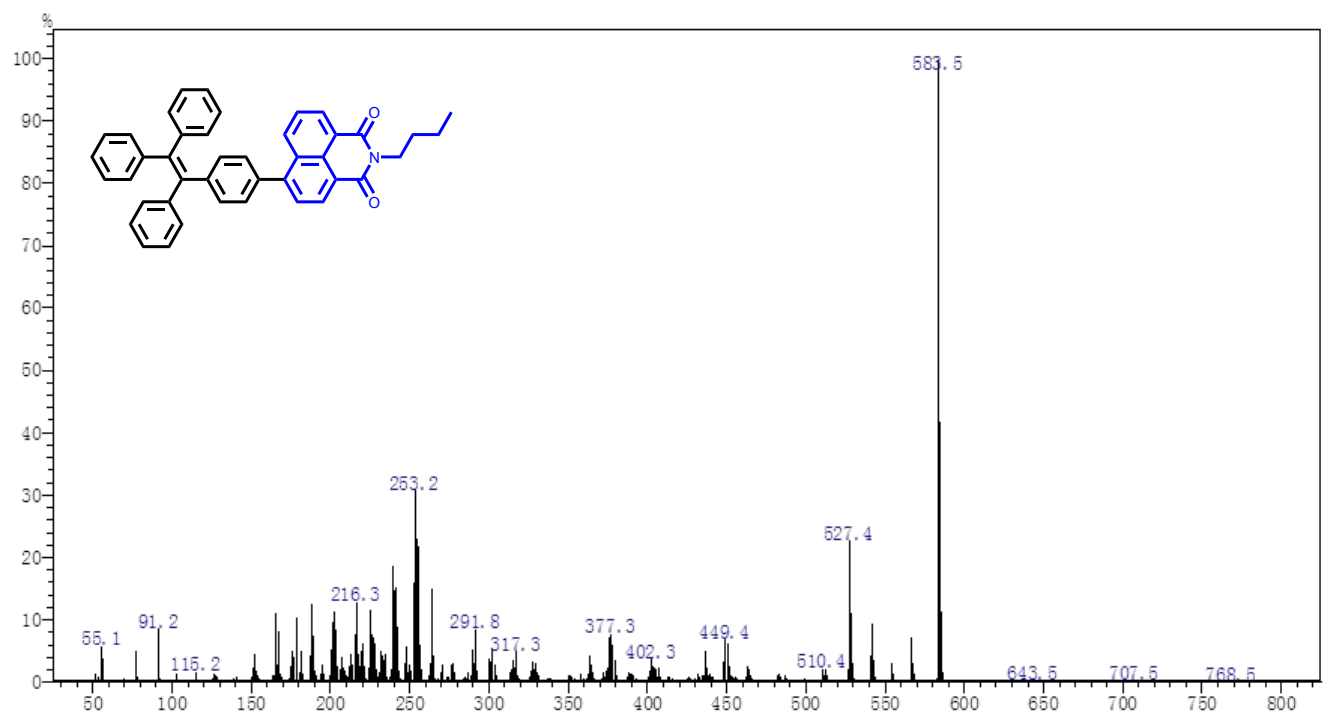

Figure S38. Mass spectrum of compound 3. 


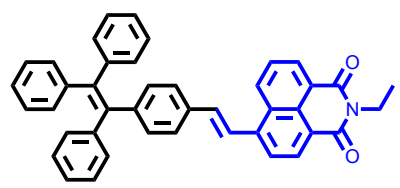

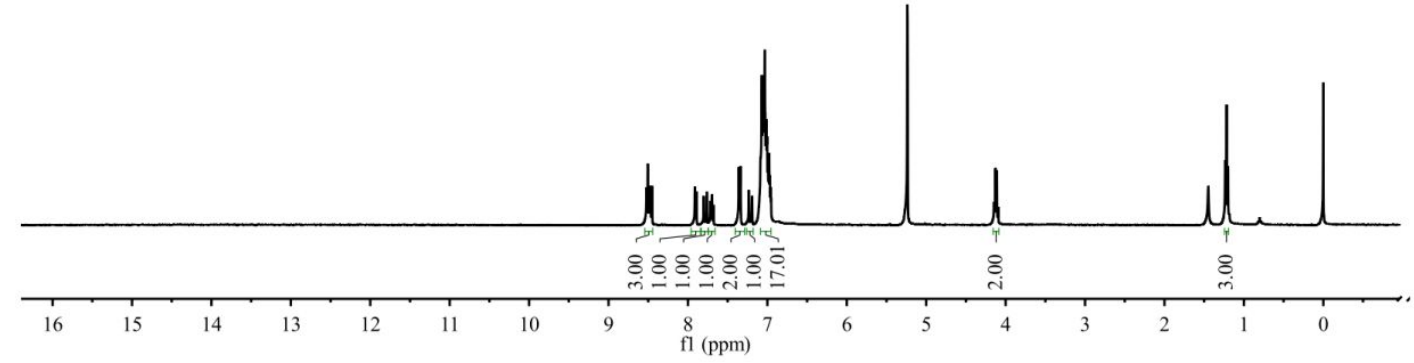

Figure S39. ${ }^{1} \mathrm{H}$ NMR spectrum of compound 4.

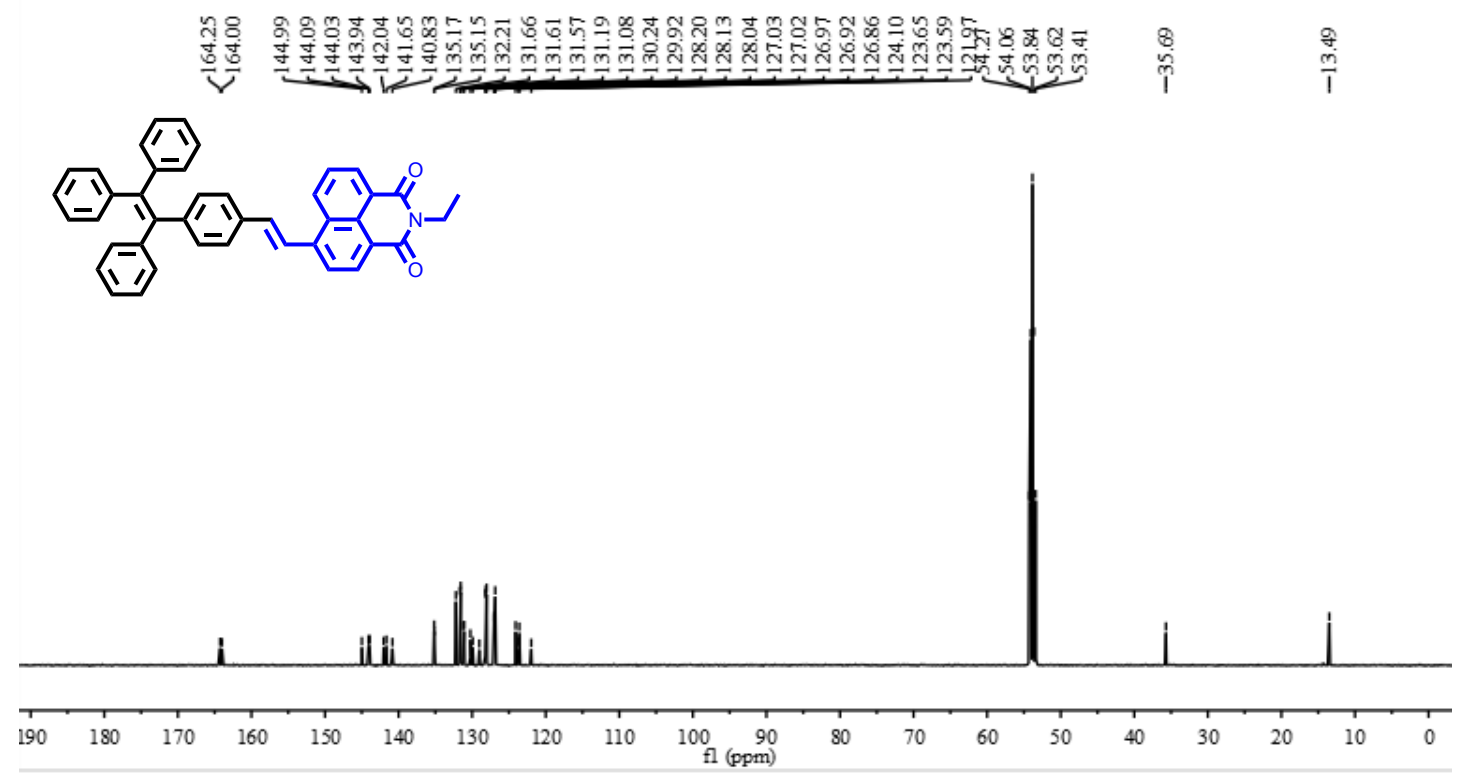

Figure S40. ${ }^{13} \mathrm{C}$ NMR spectrum of compound 4. 


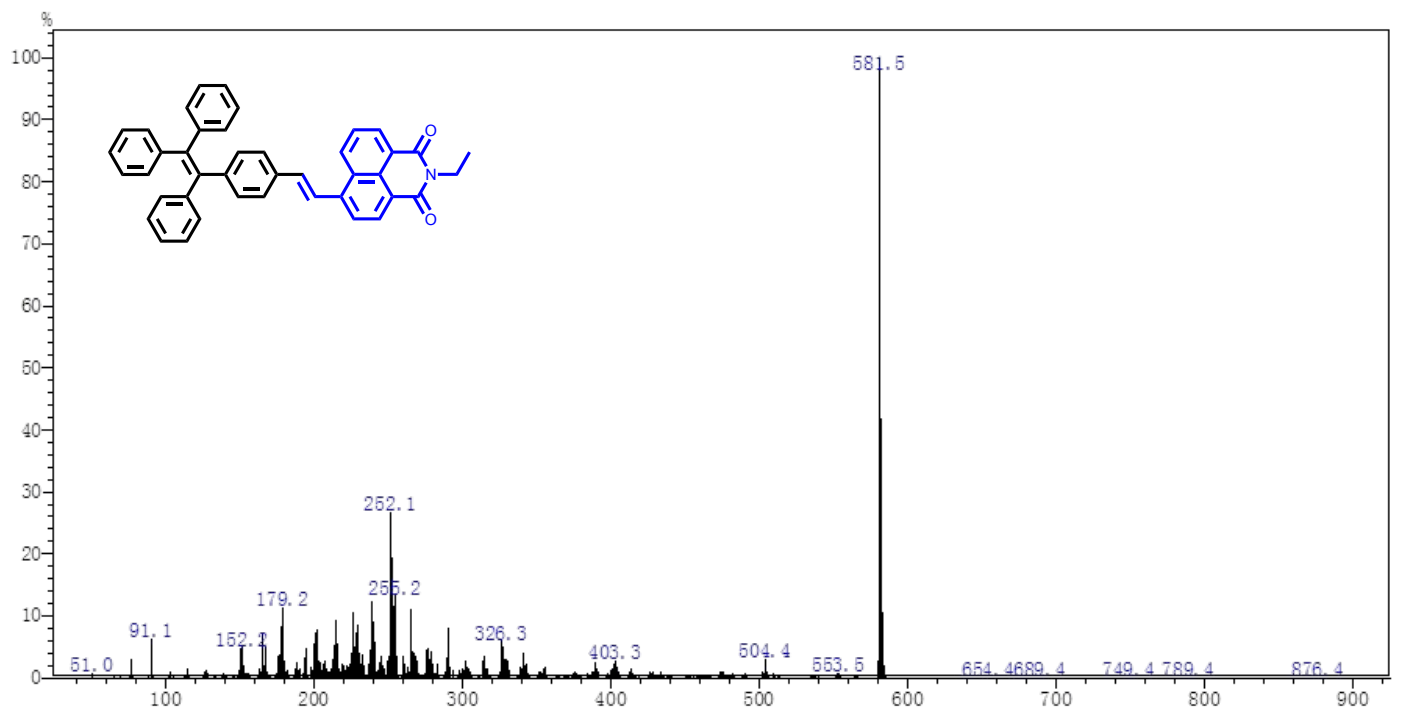

Figure S41. Mass spectrum of compound 4.

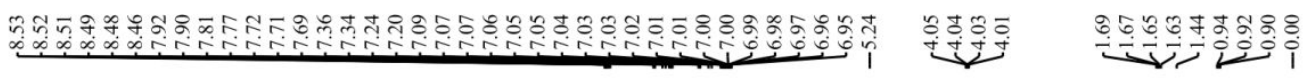

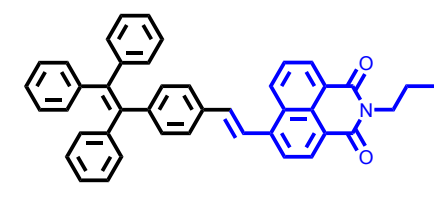

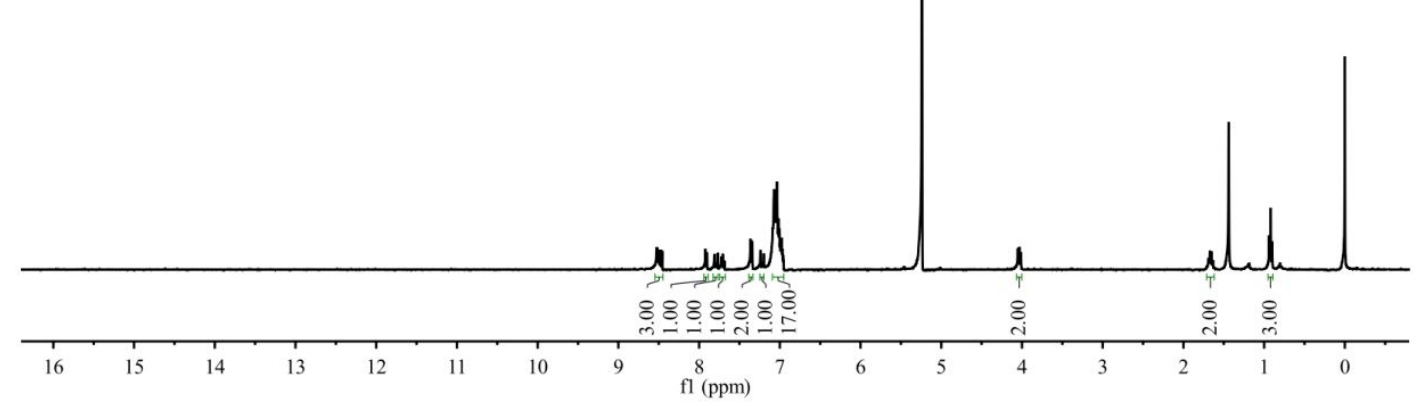

Figure S42. ${ }^{1} \mathrm{H}$ NMR spectrum of compound 5 . 


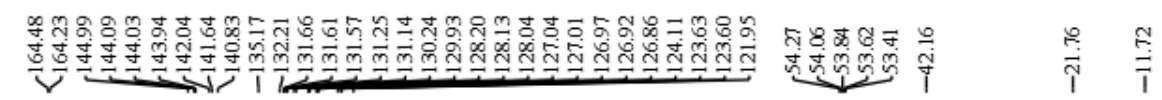
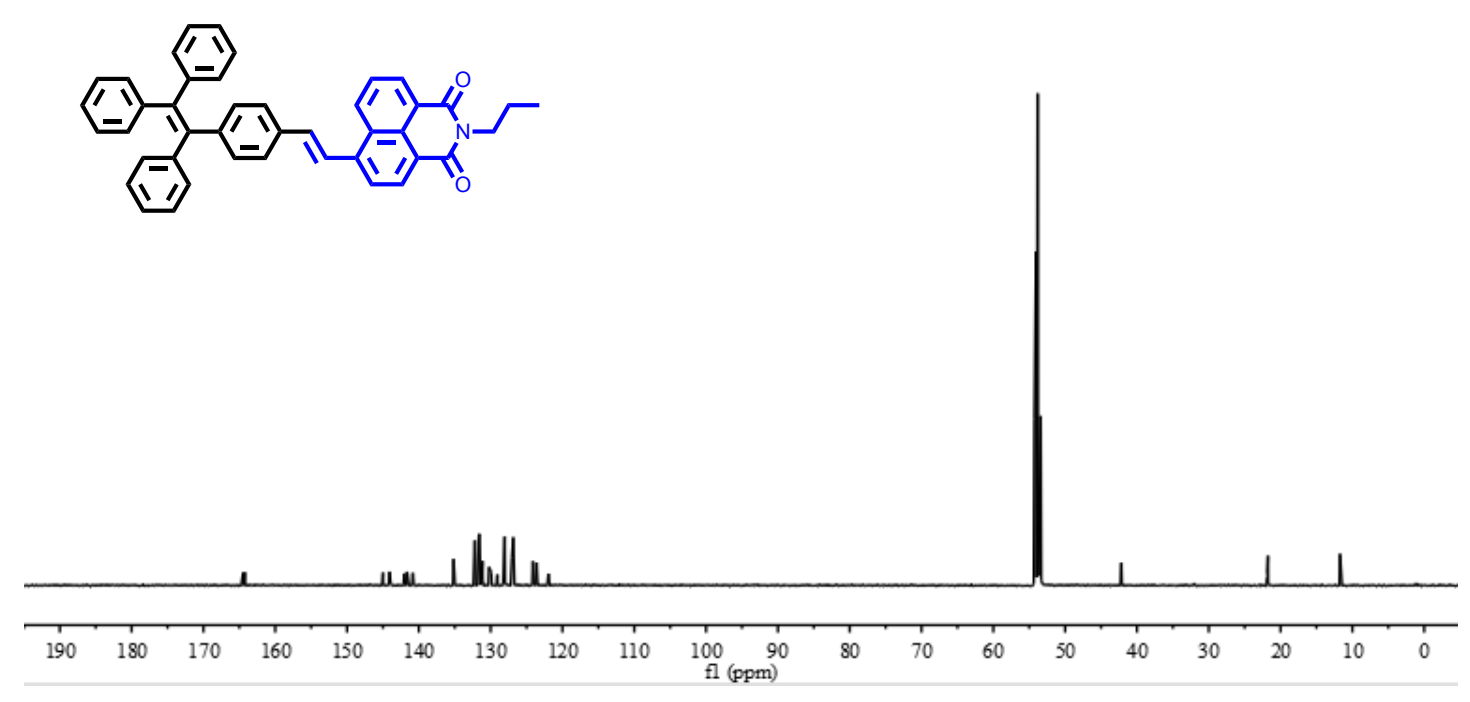

Figure S43. ${ }^{13} \mathrm{C}$ NMR spectrum of compound 5 .

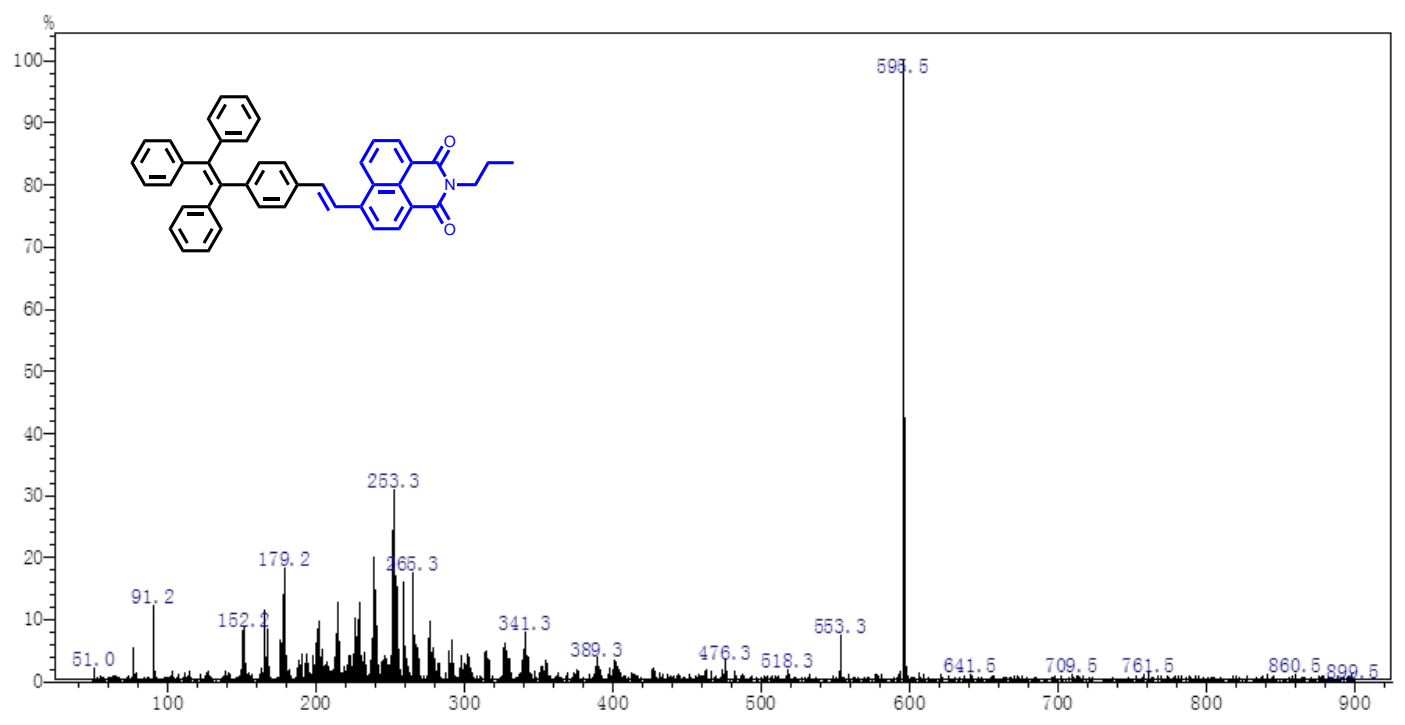

Figure S44. Mass spectrum of compound 5. 


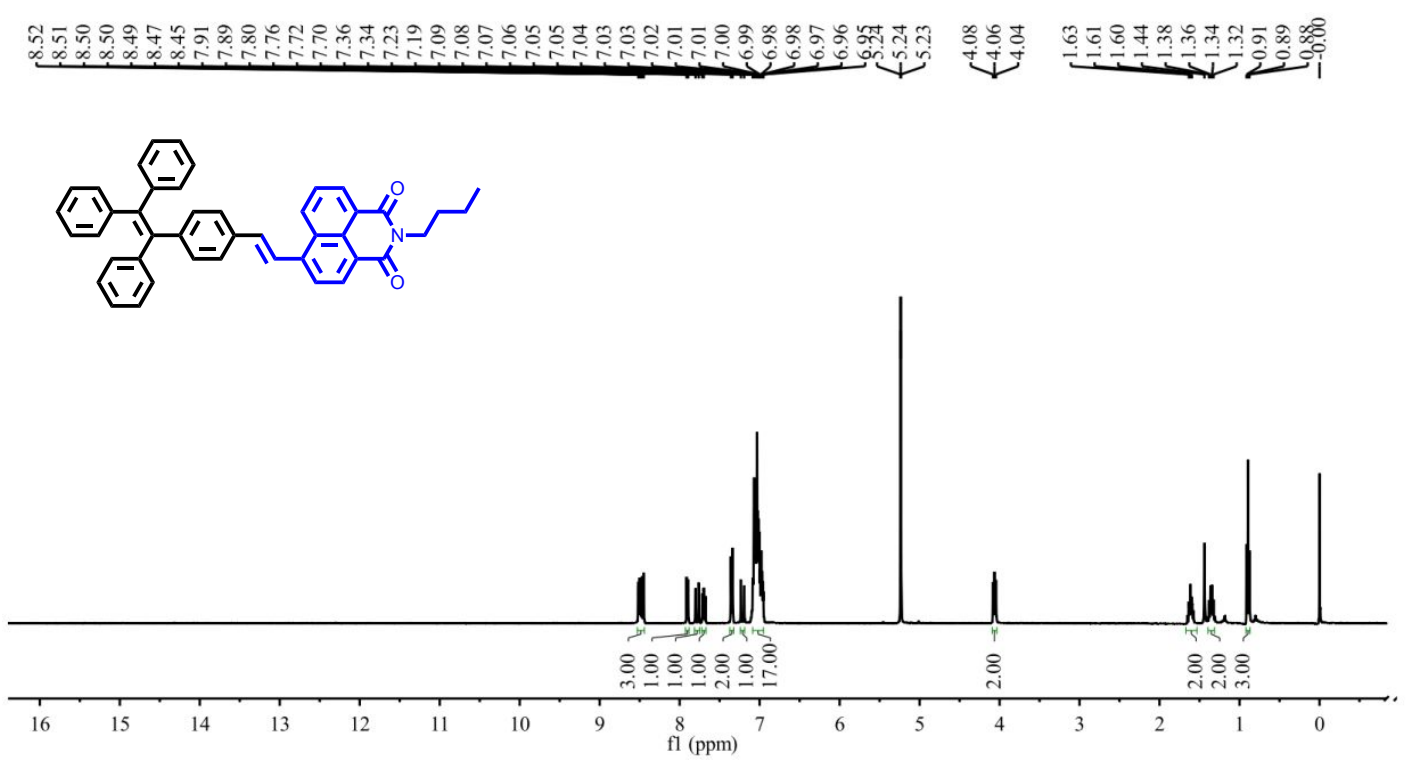

Figure S45. ${ }^{1} \mathrm{H}$ NMR spectrum of compound 6 .

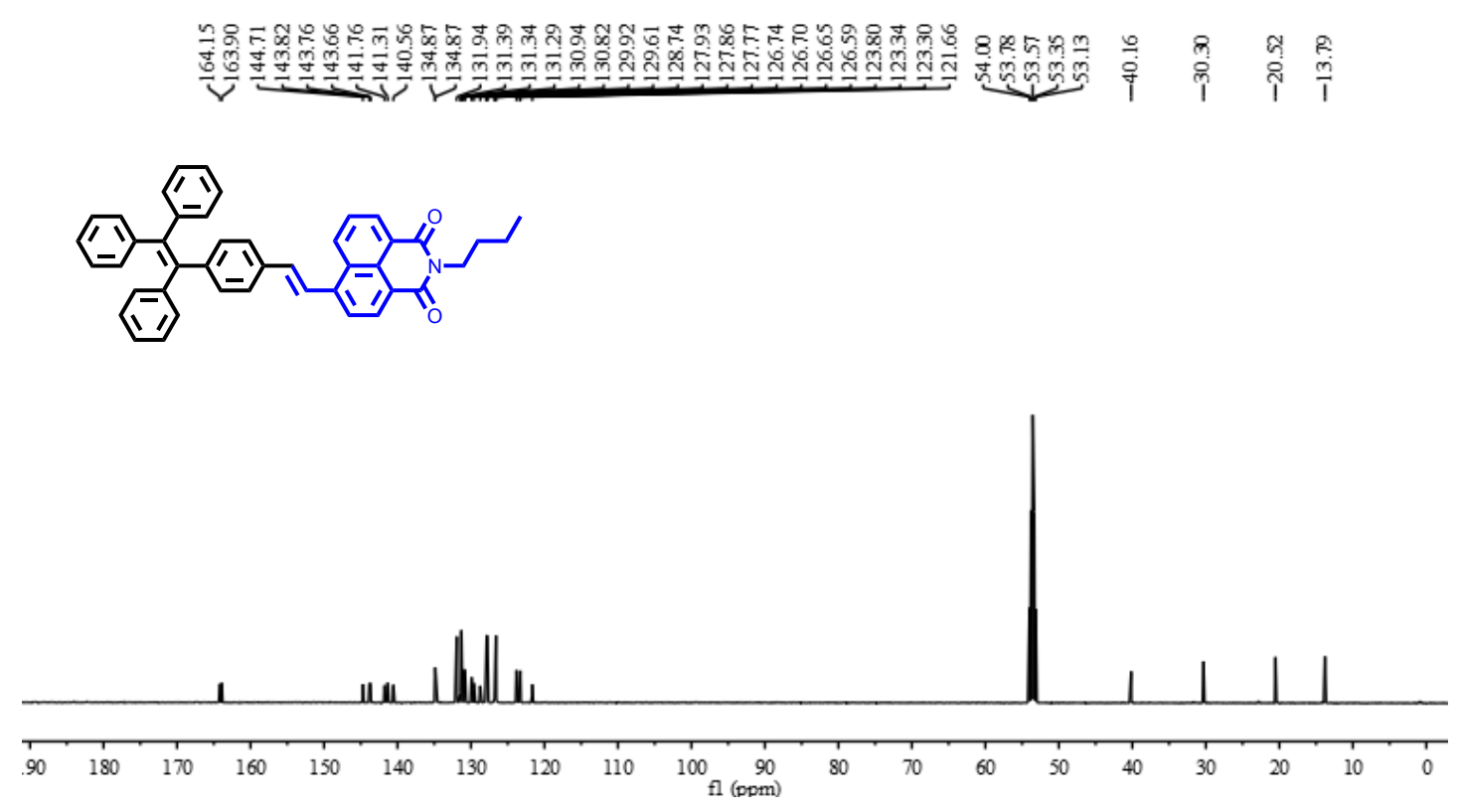

Figure S46. ${ }^{13} \mathrm{C}$ NMR spectrum of compound 6 . 


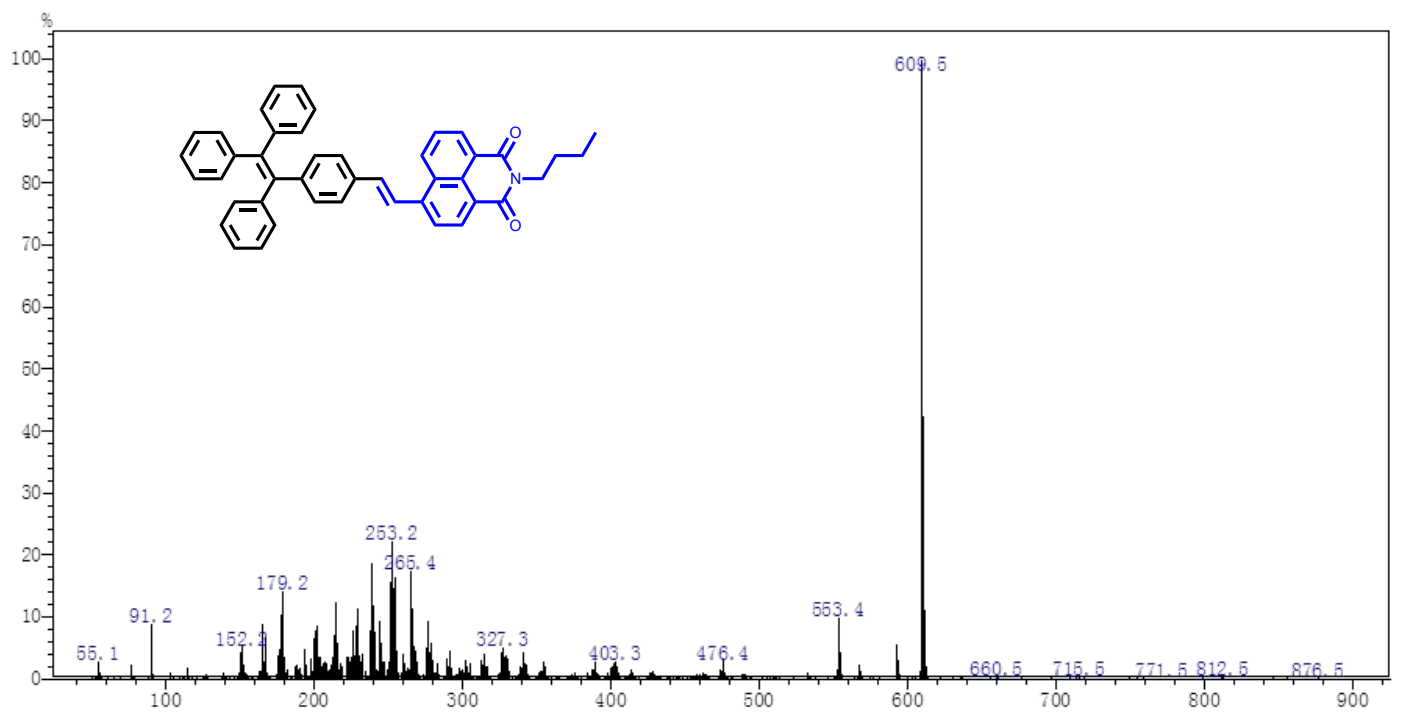

Figure S47. Mass spectrum of compound 6 . 